\title{
Strong many-particle localization and quantum computing with perpetually coupled qubits
}

\author{
L.F. Santos ${ }^{1}$, M.I. Dykman ${ }^{1 *}$, and M. Shapiro ${ }^{2}$ \\ ${ }^{1}$ Department of Physics and Astronomy, and ${ }^{2}$ Department of Mathematics, \\ Michigan State University, East Lansing, MI 48824 \\ F.M. Izrailev \\ Instituto de Física, Universidad Autónoma de Puebla, Puebla 72570, México
}

(Dated: November 13, 2018)

\begin{abstract}
We demonstrate the onset of strong on-site localization in a one-dimensional many-particle system. The localization is obtained by constructing, in an explicit form, a bounded sequence of on-site energies that eliminates resonant hopping between both nearest and remote sites. This sequence leads to quasi-exponential decay of the single-particle transition amplitude. It also leads to strong localization of stationary many-particle states in a finite-length chain. For an infinite chain, we instead study the time during which all many-particle states remain strongly localized. We show that, for any number of particles, this time exceeds the reciprocal frequency of nearest-neighbor hopping by a factor $\sim 10^{5}$ already for a moderate bandwidth of on-site energies. The proposed energy sequence is robust with respect to small errors. The formulation applies to fermions as well as perpetually coupled qubits. The results show viability of quantum computing with timeindependent qubit coupling.
\end{abstract}

PACS numbers: 03.67.Lx,72.15.Rn,75.10.Pq,73.23.-b

\section{INTRODUCTION}

Disorder-induced localization has been one of the central problems of condensed matter physics, starting with the Anderson paper [1]. It has also attracted much attention in other physical contexts, quantum chaos being an example 2, 3. . Recent interest in quantum computing has further emphasized its importance and allowed looking at it from a somewhat different perspective.

In many proposed physical implementations of a quantum computer (QC) the qubit-qubit interaction is not turned off [4, 5, 6, 7, 8, 9, 10]. The interaction may lead to excitation hopping from one qubit to another. However, control and measurement should be presumably performed on individual qubits. Therefore it is essential to prevent excitation transfer between operations. This makes localization a prerequisite for quantum computing with perpetually coupled qubits. Several approaches to quantum computing with perpetually coupled qubits have been proposed recently [11, 12, 13]

In a multi-excitation system like a system of interacting electrons or a $\mathrm{QC}$, inter-site (or inter-qubit) transitions are a many-body effect, they involve several excitations. In spite of the broad interest in the problem of many-particle localization, only a limited number of analytical results has been obtained [14]. Numerical results are also limited: classical computers do not allow studying a large number of particles, because the Hilbert space is exponentially large. On the other hand, QC's with perpetually coupled qubits provide a unique means

*e-mail: dykman@pa.msu.edu for investigating localization in a controllable setting.

In this paper we study strong on-site many-particle localization. It implies that each particle (or excitation) is nearly completely confined to one site. This is a stronger condition than just exponential decay of the wave function, and it is this condition that must be met in a QC.

A well-known argument suggests that it is hard to strongly localize a disordered many-particle system where the on-site energies are random and uniformly distributed within a finite-width band [15]. Indeed, consider a state where particles occupy $N$ sites. For short-range hopping, it is directly coupled to $\propto N$ other $N$-particle states. With probability $\propto N$ one of them will be in resonance with the initial state. For large $N$ this leads to state hybridization over time $\sim J^{-1}$, where $J$ is the inter-site hopping integral (we set $\hbar=1$ ).

In a $\mathrm{QC}$, the quantity $J$ is determined by the qubitqubit interaction and usually characterizes the rate of two-qubit operations. At the same time, the qubit energies are not random and often can be individually controlled. It is this control that makes QC's advantageous for studying many-particle localization, as it becomes possible to construct a "disordered" energy sequence site by site.

Localization can be considered from two points of view. One is based on the analysis of stationary states of a many-particle system. The other is based on studying the system dynamics. Consider a state with particles occupying a given set of sites, which is called an on-site state (or a quantum register). As a result of hopping it can hybridize with another on-site state with nearly the same energy. We will study the time it takes for resonant hybridization to happen, which we call the localization lifetime $t_{\text {loc }}$. 
In a $\mathrm{QC}$ all states have a finite coherence time due to coupling to the environment and external noise. For successful QC operation, delocalization should not occur during this time. For most of the proposed models of a QC, the coherence time is $\lesssim 10^{5} \mathrm{~J}^{-1}$. Therefore it is sufficient to have the localization lifetime $\gtrsim 10^{5} \mathrm{~J}^{-1}$. Such lifetime-based formulation of the many-particle localization problem is relevant to condensed-matter systems as well, because of finite decay and decoherence times of quasiparticles for nonzero temperatures.

Here we construct a bounded sequence of on-site energies in a 1D chain and show that it leads to a long localization lifetime. We provide evidence that it also leads to strong localization of many-particle stationary states in sections of the chain with length up to 12 sites. In a sense, this is an explicit construction of an efficiently localizing on-site disorder.

In a QC, on-site excitation energies are interlevel distances of the qubits. They can often be individually controlled, which makes it possible to construct an arbitrary energy sequence. However, since the qubit tuning range is limited, so should be the energy bandwidth. A smaller bandwidth leads also to a higher speed of quantum gate operations, particularly if they involve changing qubit energies [16]. Of course, condensed-matter systems always have bounded bandwidth as well.

In condensed-matter physics, localization by "controlled" disorder has been studied in depth and many interesting results have been obtained in the context of incommensurate periodic potentials, see Refs. 17, 18, 19 and papers cited therein. In contrast to this work, we are interested in strong on-site localization, and not only for single-, but in the first place, for many-particle states. The "potential" that we propose is not quasiperiodic, and our analytical techniques, including the time-dependent formulation for many-body systems, are different from the methods developed for quasi-periodic potentials.

To strongly localize one particle, the difference between excitation energies on neighboring sites should be much larger than the hopping integral $J$. However, even for nearest neighbor coupling, the energies of remote sites should also differ to prevent transitions between them via intermediate (nonresonant) sites. The further away the sites are, the smaller their energy difference can be. This idea is implemented in our energy sequence. As a consequence, the single-particle transition amplitude displays nearly exponential decay with distance. We show that the decay exponent weakly depends on site and find rigorous bounds on its value.

For many-particle localization one has to suppress not only single-particle, but also combined resonances, which involve simultaneous transitions of several interacting excitations. There is no known way to eliminate all such resonances in an infinite system. However, with the increasing "order" of the transition, i.e., the number of involved excitations and/or intermediate virtual states, the effective hopping integral may quickly fall off, lead- ing to an increase of the transition time. Then to obtain a desired lifetime of a localized state it is sufficient to eliminate resonances up to a certain order. We explicitly show how to do it up to fifth order, for our sequence.

In a real system it will be possible to tune the energies only with certain precision. We study how errors in the energies affect localization and show that our sequence is stable with respect to small errors. We also demonstrate that, in terms of strong on-site localization, even for a small chain the constructed energy sequence is much better than fully random on-site energies with the same overall bandwidth.

The paper is organized as follows. In Sec. II we discuss the Hamiltonian of coupled qubits and introduce a physically motivated one-parameter sequence of on-site energies. In Sec. III one-particle localization of stationary states is studied and quasi-exponential decay of the transition amplitude is demonstrated. A rigorous proof of such decay is provided in Appendix A. The relevant scaling properties are analyzed in Appendix B. In Sec. IV the inverse participation ratio is calculated for many-particle states in a section of a $1 \mathrm{D}$ chain. This ratio can be made very close to one in a broad range of the parameter of the on-site energy sequence, but it also displays sharp resonant peaks as a function of this parameter. These peaks are discussed in Appendix C. The lifetime of localized states is considered in Sec. V. A minor modification of the energy sequence allows one to open a gap in the spectrum of combined many-excitation transitions up to 5th order, which is sufficient for extremely long localization lifetime. The role of errors in the on-site energies is studied, and robustness of the results with respect to these errors is demonstrated in Sec. VI. In Sec. VII a highly symmetric period-doubling sequence of on-site energies is analyzed along with a sequence of uncorrelated on-site energies. Both are by far inferior, in terms of localization, to the sequence discussed in Secs. II-V. Sec. VIII contains concluding remarks.

\section{THE MODEL}

The problem of localization can be formulated in a similar way for one-dimensional systems of interacting fermions and spins. The formulation also applies to qubits during the time when no gate operations and measurements are performed, i.e., there are no timedependent fields that would modulate the qubits.

The relation between spin and qubit systems is simple: qubits are two-level systems, and therefore can be described by $S=1 / 2$ spins in a magnetic field. Then the excitation energy of a qubit becomes the Zeeman energy of a spin, whereas the qubit-qubit interaction becomes the exchange spin coupling. Note that the physical interaction itself may be of a totally different nature, e.g., electric dipolar or quadrupolar.

For many proposed realizations of QC's the qubit excitation energies are large compared to the interaction. 
Then the qubit-qubit interaction is described by the spincoupling Hamiltonian of the form

$$
H_{S}=\frac{1}{2} \sum_{n, m}^{\prime}\left[J_{n m}^{x x}\left(S_{n}^{x} S_{m}^{x}+S_{n}^{y} S_{m}^{y}\right)+J_{n m}^{z z} S_{n}^{z} S_{m}^{z}\right] .
$$

Here, $n, m$ enumerate sites in the $1 \mathrm{D}$ spin chain, $z$ is the direction of the effective magnetic field, and $J_{n m}^{\mu \mu}$ are the interaction parameters $(\mu=x, y, z)$. In mapping the qubit interaction on the exchange coupling we kept only those terms which, in the Heisenberg representation, do not oscillate at qubit transition frequencies. This is why we have set $J_{n m}^{x x}=J_{n m}^{y y}$. In the case of qubits, the terms $S_{n}^{x} S_{m}^{x}+S_{n}^{y} S_{m}^{y} \equiv(1 / 2)\left(S_{n}^{+} S_{m}^{-}+S_{n}^{-} S_{m}^{+}\right)$lead to excitation transfer between the qubits $n, m$ provided their energies are close. We note that the spin interaction of the form (11) conserves the number of excitations in the system.

It is convenient to map the spin system onto a system of spinless fermions via the Jordan-Wigner transformation [21]. For nearest neighbor coupling, the Hamiltonian of the fermion system becomes

$$
\begin{aligned}
& H=H_{0}+H_{i}, \\
& H_{0}=\sum_{n} \varepsilon_{n} a_{n}^{\dagger} a_{n}+\frac{1}{2} J \sum_{n}\left(a_{n}^{\dagger} a_{n+1}+a_{n+1}^{\dagger} a_{n}\right), \\
& H_{i}=J \Delta \sum_{n} a_{n}^{\dagger} a_{n+1}^{\dagger} a_{n+1} a_{n} .
\end{aligned}
$$

Here, $a_{n}^{\dagger}, a_{n}$ are the fermion creation and annihilation operators; the presence of a fermion on site $n$ corresponds to the $n$th spin being excited. The parameter $J=J_{n n+1}^{x x}$ is the fermion hopping integral. The parameter $J \Delta=J_{n n+1}^{z z}$ gives the interaction energy of fermions on neighboring sites. If the coupling of the underlying spins is isotropic, we have $\Delta=1$. The on-site fermion energies $\varepsilon_{n}$ are the Zeeman energies of the spins (excitation energies of the qubits) counted off from the characteristic central energy which is the same for all spins inside the chain. For concreteness we set $J, \Delta>0$.

Localization, and in particular weak localization, is often described in terms of the decay of the wave functions of stationary states at large distances. In contrast, here we are interested in strong on-site localization. It is determined by the short-range behavior of stationary states and corresponds to confinement of each particle to one or maybe a few neighboring sites.

Strong localization can be conveniently characterized by the inverse participation ratio (IPR), which shows over how many sites the wave function spreads. For an $N$ particle wave function $\left|\psi_{N \lambda}\right\rangle$ ( $\lambda$ enumerates the stationary states) the IPR is given by the expression

$$
I_{N \lambda}=\left(\sum_{n_{1}<\ldots<n_{N}}\left|\left\langle 0\left|a_{n_{N}} a_{n_{N-1}} \ldots a_{n_{1}}\right| \psi_{N \lambda}\right\rangle\right|^{4}\right)^{-1}
$$

where $|0\rangle$ is the vacuum state. In what follows we will sometimes use the notation $\left|\Phi\left(k_{1}, k_{2}, \ldots\right)\right\rangle=$ $a_{k_{1}}^{\dagger} a_{k_{2}}^{\dagger} \ldots|0\rangle$ for the on-site wave function (quantum register) in which sites $k_{1}, k_{2}, \ldots$ are occupied and other sites are empty. The quantity (3) is also sometimes called the number of participating components, it shows how many matrix elements $\left\langle\Phi\left(k_{1}, k_{2}, \ldots\right) \mid \psi_{N \lambda}\right\rangle$ are substantial, i.e., of order of their maximal value with respect to $k_{1}, k_{2}, \ldots$.

For fully localized states $I_{N \lambda}=1$. Strong on-site localization means that $I_{N \lambda}$ is close to 1 for all states $\lambda$. In this case both the average IPR

$$
\left\langle I_{N}\right\rangle=C_{N \lambda}^{-1} \sum_{\lambda} I_{N \lambda}
$$

and $I_{N \max }=\max _{\lambda} I_{N \lambda}$ are close to one. Here, $C_{N \lambda}$ is the total number of $N$-particle states; for an $L$-site chain $C_{N \lambda}=L ! / N !(L-N)$ !. Smallness of $\left\langle I_{N}\right\rangle-1$ is a weaker condition, it is an indication of strong localization of most of the states.

In the opposite limit of extended states we have $\left\langle I_{N \lambda}\right\rangle \sim C_{N \lambda} \gg 1$. A simple example is the case of one particle in an open chain (a chain with free boundaries) with $\varepsilon_{n}=$ const. The wave functions of the particle are sinusoidal, and for an $L$-site chain $\left\langle I_{1}\right\rangle=2(L+1) / 3$. The mean IPR sharply increases with the number of particles $N$, for $N \leq L / 2$.

\section{A. The on-site energy sequence}

Localization requires that the on-site energies $\varepsilon_{n}$ be tuned away from each other. The strategy for choosing the sequence of $\varepsilon_{n}$ while keeping the overall bandwidth of the energy spectrum finite can be as follows. First, we separate the energies of nearest neighbors by splitting $\varepsilon_{n}$ into two subbands, with even and odd $n$, respectively. The distance $h$ between the subbands should significantly exceed the hopping integral $J$. Then each subband is further split into two subbands in order to detune next nearest neighbors. The splitting between these subbands can be less than $h$. This is because next-nearest-neighbor hopping occurs via virtual transitions to a nonresonant nearest-neighbor site, and therefore the effective hopping integral is $\sim J^{2} / h$. The procedure of band splitting should be continued, and higher-order splitting can be smaller and smaller.

We now introduce a simple sequence of $\varepsilon_{n}$ that implements the structure described above. Except for the energy scaling factor $h$, this sequence is characterized by one dimensionless parameter $\alpha$. As we show, it can already be efficient in terms of strong localization. For a semi-infinite chain with $n \geq 1$ we set

$$
\varepsilon_{n}=\frac{1}{2} h\left[(-1)^{n}-\sum_{k=2}^{n+1}(-1)^{\lfloor n / k\rfloor} \alpha^{k-1}\right],
$$

$(\lfloor\cdot\rfloor$ is the integer part).

Sequence (4) does not have any simple symmetry. For example, it is not self-similar: the subband widths do not scale with the distance between the sites that belong to the same subband (cf. Sec. VII). Nor is sequence (41) quasi-periodic. However, the coefficients at any given power $\alpha^{q}$ are repeated with period 2(q+1). This important property is essential for obtaining analytical results, see Appendix A. 
The energy spectrum (4) is illustrated in Fig. 11 The left panel gives the energies of the first 50 sites. It is seen that the sites with close $\varepsilon_{n}$ are spatially separated, whereas the sites with close $n$ are separated energetically. The multisubband structure of the spectrum is clearly seen in the right panel. For small $\alpha$, the two major subbands have width $\approx \alpha h$ and are separated by $\approx h$. The splitting of higher-order subbands is proportional to higher powers of $\alpha$. As $\alpha$ increases the subbands start overlapping, and for $\alpha \gtrsim 0.4$ separation between the subbands disappears.

As a result of the low symmetry, different subbands in the right panel of Fig. 1 have different numbers of points, i.e., $\varepsilon_{n}$ are not evenly distributed among the subbands. This turns out to be important for strong many-particle localization. The case of a symmetric sequence is discussed in Sec. VII.
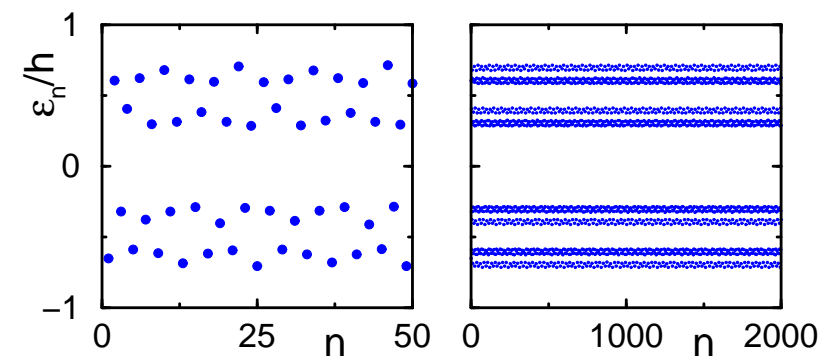

FIG. 1: (color online) The energies $\varepsilon_{n} / h$ for $\alpha=0.3$. The left panel shows $\varepsilon_{n} / h$ for the sites $n=1,2, \ldots, 50$. Sites with close on-site energies are spatially separated. The right panel shows $\varepsilon_{n} / h$ for a much longer array, $n=1, \ldots, 2000$. The energy spectrum displays a multisubband structure, with clearly identifiable 16 subbands in this case.

An important advantageous feature of sequence (4) for quantum computing is that it is convenient for performing gate operations. For single-qubit gates, a single particular radiation frequency can be used to resonantly excite different qubits. It has to be chosen near the average single-qubit transition frequency (which corresponds to $\varepsilon=0)$. Then qubits can be selectively excited by tuning them to this frequency. The transition frequency of the qubit depends on whether neighboring qubits are excited. This can be used for implementing a CNOT gate. Alternatively, neighboring qubits can be tuned in resonance with each other, which will lead to a two-qubit excitation swap [16].

\section{SINGLE-PARTICLE LOCALIZATION: STATIONARY STATES}

\section{A. The transition amplitude}

In a $1 \mathrm{D}$ system with random on-site energies all singleparticle stationary states are localized, even for weak disorder, and exponentially decay at large distances. Al- though sequence (4) is not random, the transition amplitudes also display quasi-exponential decay at large distances provided $J \ll \alpha h$, as follows from the results of Appendix A. In this paper we are primarily interested in the short-range behavior. It turns out that a particle is confined much stronger in the case of sequence (4) than in the case of random on-site energies distributed within the same energy band, see Sec. VII. The confinement quickly strengthens with the increasing parameter $\alpha$ once $\alpha$ exceeds a certain threshold value $\alpha_{\mathrm{th}}$.

Spatial decay of single-particle stationary states can be characterized by the amplitude of a particle transition from site $n$ to site $n+m$. To the lowest order in $J / h$ it has the form

$$
K_{n}(m)=\prod_{k=1}^{m} J /\left|2\left(\varepsilon_{n}-\varepsilon_{n+k}\right)\right| .
$$

For sequence (4) in the limit of small $\alpha$ the energy difference $\left|\varepsilon_{n+m}-\varepsilon_{n}\right|$ can be approximated by its leading term, so it is $\sim h$ for odd $m$ and $\sim \alpha h$ for odd $m / 2$. In general, the larger is $m$ the higher may be the order in $\alpha$ of the leading term in $\left|\varepsilon_{n+m}-\varepsilon_{n}\right|$.

The asymptotic behavior of the function $K_{n}(m)$ for small $\alpha$ and large $m$ can be studied rigorously. The analysis is based on some results of number theory. It is given in Appendix A. It shows that $K_{n}(m)$ decays with the distance $m$ quasi-exponentially,

$$
K_{n}(m)=\alpha^{-\nu|m|}(J / 2 h)^{|m|} .
$$

The exponent $\nu$ is determined by $\lim \log K_{n}(m) / m$ for $m \rightarrow \infty$ and depends on $n$. The values of $\nu$ are bound to a narrow region centered at $\nu=1$, with $0.89<\nu<1.19$. For estimates one can use $\nu=1$, i.e.,

$$
K_{n}(m) \approx K^{|m|}, \quad K=J / 2 \alpha h .
$$

The decay length of the transition amplitude is then $1 /|\ln K|$.

The numerical values of $\nu$ for different $n$ and $m$ are shown in Fig. 2] They were obtained by keeping the leading term with respect to $\alpha$ in each energy difference $\varepsilon_{n}-\varepsilon_{n+k}$ with $1 \leq k \leq m$. The data are in excellent agreement with the asymptotic theory.

We have also studied decay in the "opposite" direction, i.e., for negative $m$ in Eq. (5) (however, $m+n \geq 1$ ). The asymptotic expression (6) applies in this case, provided $n,|m| \gg 1$. The numerical data also agree with the theory.

Equation (6) gives the tail of the transition amplitude for $J / 2 h \alpha \ll 1$. It does not immediately describe strong single-particle localization, which is determined by the short-range behavior of the wave function. However, one may expect that strong localization should occur when $\alpha$ becomes much larger than a typical threshold value $\alpha_{\text {th }}=$ $J / 2 h$. In fact, Eq. (6) describes the transition amplitude only when $\alpha_{\text {th }} \ll \alpha \ll 1$. We note that the inequality $\alpha_{\text {th }}<0.4$, which is necessary to avoid overlapping of the subbands, is satisfied already when the ratio of the energy bandwidth to the hopping integral $h / J$ exceeds the comparatively small value 1.3 . 


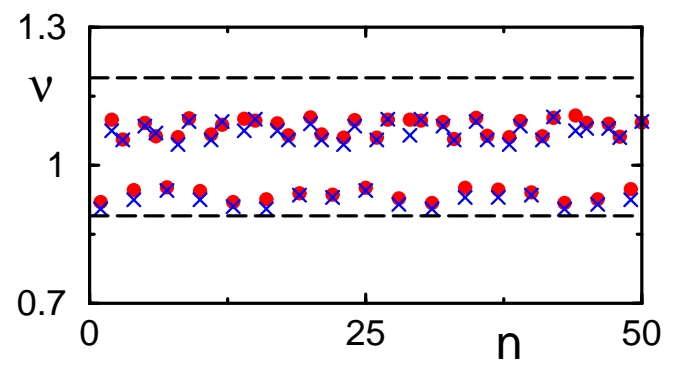

FIG. 2: (color online). The exponent $\nu$ of the $\alpha$-dependence of the transition amplitude $K_{n}(m)$ for the efficient distance $m=200$ (crosses) and $m=1000$ (circles) as a function of site number $n$. The dashed lines show the analytical limits on $\nu$.

\section{B. The inverse participation ratio}

A quantitative indication of strong localization of single-particle stationary states is that $I_{1 \lambda}-1 \ll 1$ for all states $\lambda$. Numerical results on $\left\langle I_{1}\right\rangle$ and $I_{1}$ max as functions of the energy spectrum parameter $\alpha$ for two values of the scaled bandwidth $h / J$ are shown in Fig. [3. They were obtained by diagonalizing the Hamiltonian (2) numerically. The data refer to open chains of three different lengths $L$, with the first site being always $n=1$ in Eqs. (2), (4). The sum over $n$ in the terms $\propto J, J \Delta$ in Eq. (2) ran from $n=1$ to $n=L-1$.

In the fermion Hamiltonian (2), the energies $\varepsilon_{n}$ differ from the Zeeman energies of spins by $-J \Delta$ inside the chain. On the boundaries this shift is $-J \Delta / 2$. To make the Zeeman energies equal to $\varepsilon_{n}+$ const, with the constant being the same for all spins, we added the term $-\frac{1}{2} J \Delta\left(S_{1}^{z}+S_{L}^{z}\right)$ to the spin Hamiltonian (1). Then the numerical results for a finite open chain equally apply to both spin and fermion systems.

In the limit $\alpha \rightarrow 0$, the energies of single-particle stationary states form two bands centered at $h / 2$ for even $n$ and $-h / 2$ for odd $n$. For $h \gg J$ the widths of these bands are $\sim J^{2} / h$. The system is equivalent to two weakly coupled translationally-symmetric open chains; the band wave functions are sinusoidal, which gives $\left\langle I_{1}\right\rangle=(L+2) / 3$. This agrees with the value of $\left\langle I_{1}\right\rangle$ for $\alpha \rightarrow 0$ in Fig. 3

For nonzero $\alpha$ the on-site level detuning (4) breaks translational symmetry. As $\alpha$ increases, the bands at $\pm h / 2$ are split, and more and more subbands are resolved in the energy spectrum. Respectively, $\left\langle I_{1}\right\rangle$ decreases. It sharply drops to $\approx 1$ in a narrow region, which can be conditionally associated with a transition to strong localization. The center of the transition region gives the threshold value $\alpha_{\text {th }}$ of the parameter $\alpha$. It appears to be independent of the chain length $L$. The estimate $\alpha_{\text {th }}=J / 2 h$ from Eq. (6) is in good agreement with the numerical data for different $h / J$.

When $\alpha_{\text {th }} \ll \alpha \ll 1$, all stationary states are strongly localized. Tails of the wave functions are small and lim-

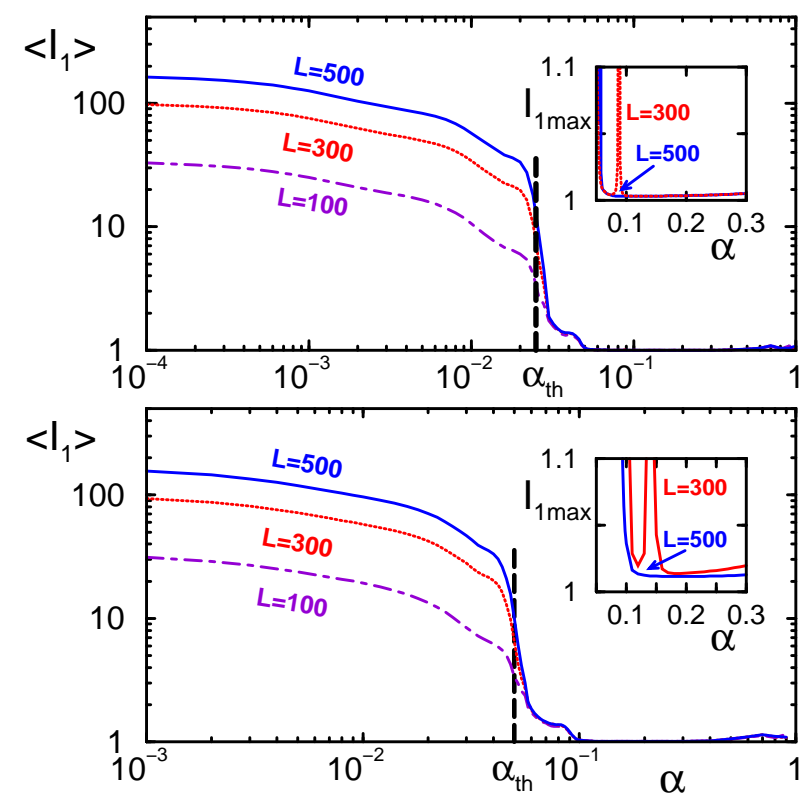

FIG. 3: (color online). The mean single-particle inverse participation ratio $\left\langle I_{1}\right\rangle$ vs. $\alpha$ for $h / J=20$ (upper panel) and $h / J=10$ (lower panel). The data refer to three values of the chain length $L$. The vertical dashed lines show the analytical estimate for the threshold of strong localization. The insets show the maximal IPR over all eigenstates, $I_{1 \max } \equiv \max _{\lambda} I_{1 \lambda}$. It sharply decreases with the increasing $\alpha$. The peak of $I_{1} \max$ for $L=300$ near $\alpha=0.1$ is due to the boundary. Near the minimum over $\alpha$, we have $I_{1 \max } \approx 1.01$ for $h / J=10$, and $I_{1 \max } \approx 1.003$ for $h / J=20$. This demonstrates strong single-particle localization.

ited mostly to nearest neighbors, which leads to

$$
I_{1 \lambda}-1 \approx J^{2} / h^{2}
$$

to lowest order in $J / h, \alpha$.

For $\alpha \gtrsim 0.4$ the IPR increases above its minimal value. This happens because the major bands of $\varepsilon_{n}$ centered at $\pm h / 2$ start overlapping.

The minimum of the IPR over $\alpha$ is broad for large $h / J$. Near the minimum the numerical data in Fig. 3 are in good agreement with the estimate (7). The agreement becomes better with increasing $h / J$.

The insets in Fig. 3 show that the IPR as a function of $\alpha$ can have narrow resonant peaks. In the presented data they occur for the chain of length $L=300$. The peaks are seen only in $I_{1 \text { max }}$, whereas $\left\langle I_{1}\right\rangle$ remains close to 1 . This indicates that only a few on-site states are hybridized with each other.

The underlying resonance results from a different hopping-induced shift of the energy levels at the chain edges compared to the bulk. The analysis of the wave functions shows that the peak corresponds to a resonance between sites 300 and 296. Because of the hopping, the energy of site 300 is shifted by $\approx(J / 2)^{2} / h$, whereas for 
site 296 this shift is $\approx J^{2} / 2 h$. The difference of $\varepsilon_{n}$ for these sites is $\sim h \alpha^{3}$ for $\alpha \ll 1$. Then the peak should occur at $\alpha \approx(J / 2 h)^{2 / 3}$, in good agreement with the data. The effective hopping integral between the two sites is determined by virtual transitions via intermediate sites, it is $\sim J^{4} / 16 \alpha h^{3}$. Therefore the width of the peak with respect to $\alpha$ should be $\propto(J / h)^{2}$, also in agreement with the data.

In Appendix B we outline another way of looking at the effect of the parameter $\alpha$ on localization. Specifically, we study the scaling relations between $\alpha$ and $J / h$ that follow from the condition that the IPR takes on a given value.

\section{MANY PARTICLE LOCALIZATION: STATIONARY STATES}

\section{A. Many-particle hopping}

The many-particle localization problem is more complicated than the single-particle one. When the parameter of the inter-particle interaction $\Delta \neq 0$ (i) the energy levels $\varepsilon_{n}$ are shifted depending on the occupation of neighboring states, $\varepsilon_{n} \rightarrow \tilde{\varepsilon}_{n}$, (ii) there occur combinational many-particle resonances $\tilde{\varepsilon}_{n_{1}}+\ldots+\tilde{\varepsilon}_{n_{k}} \approx \tilde{\varepsilon}_{m_{1}}+$ $\ldots+\tilde{\varepsilon}_{m_{k}}$, and as a result, (iii) there occur interactioninduced many-particle transitions that may be resonant even though single-particle resonances have been suppressed. Such transitions may lead to delocalization.

In contrast, the case $\Delta=0$ corresponds to the $X Y$ type coupling between the underlying spins. In this case the single-particle results apply to the many-particle system.

To analyze many-particle effects, it is convenient to change from $a_{n}^{\dagger}, a_{n}$ to new creation and annihilation operators $b_{n}^{\dagger}, b_{n}$ that diagonalize the single-particle part $H_{0}$ of the Hamiltonian (2), $a_{n}=\sum_{k} U_{n k} b_{k}$. The unitary matrix $\hat{U}$ is the solution of the equation

$$
\begin{aligned}
& \left(U^{\dagger} H_{0} U\right)_{n m}=\varepsilon_{n}^{\prime} \delta_{n m}, \\
& \left(H_{0}\right)_{n m}=\varepsilon_{n} \delta_{n m}+\frac{1}{2} J\left(\delta_{n, m+1}+\delta_{n+1, m}\right) .
\end{aligned}
$$

Here, $\varepsilon_{n}^{\prime}$ are the exact single-particle energies,

$$
U^{\dagger} H_{0} U=\sum_{n} \varepsilon_{n}^{\prime} b_{n}^{\dagger} b_{n}
$$

For $\alpha \gg \alpha_{\text {th }}$ and $J \ll h$, when single-particle states are strongly localized, the energies $\varepsilon_{n}^{\prime}$ are close to the on-site energies $\varepsilon_{n}$. To leading order in $J / h, \alpha$ we have

$$
\varepsilon_{n}^{\prime}-\varepsilon_{n} \approx \frac{J^{2}}{2 h}\left[(-1)^{n}+\frac{1}{2}(-1)^{\lfloor n / 2\rfloor} \alpha\right] .
$$

The major term in the right-hand side corresponds simply to renormalization of the characteristic bandwidth of on-site energies $h \rightarrow h+J^{2} / 2 h$.
In terms of the operators $b_{n}, b_{n}^{\dagger}$ the interaction part of the Hamiltonian is

$$
U^{\dagger} H_{i} U=J \Delta \sum V_{k_{1} k_{2} k_{3} k_{4}} b_{k_{1}}^{\dagger} b_{k_{2}}^{\dagger} b_{k_{3}} b_{k_{4}},
$$

where the sum runs over $k_{1,2,3,4}$, and

$$
V_{k_{1} k_{2} k_{3} k_{4}}=\sum_{p} U_{p k_{1}}^{*} U_{p+1 k_{2}}^{*} U_{p+1 k_{3}} U_{p k_{4}}
$$

The Hamiltonian (11) describes the interaction of the exact single-particle excitations.

If the single-particle states are all strongly localized, the off-diagonal matrix elements $U_{n k}$ are small. They are determined by the decay of the wave functions, and therefore fall off exponentially with increasing $|k-n|$. From Eq. (6) we have $U_{n k} \sim K^{|k-n|}$ for $|k-n| \gg 1$. At the same time, the diagonal matrix element is $U_{n n} \approx 1$.

Therefore, for strong single-particle localization the major terms in the matrix $V_{k_{1} k_{2} k_{3} k_{4}}$ are those with $\varkappa=0$, where

$$
\varkappa=\min _{p}\left(\left|k_{1}-p\right|+\left|k_{2}-p-1\right|+\left|k_{3}-p-1\right|+\left|k_{4}-p\right|\right) .
$$

These terms lead to an energy shift $\propto J \Delta$ of the states depending on the number of particles on neighboring sites.

The meaning of the parameter $\varkappa$ (13) can be understood by noticing that the terms $\propto V_{k_{1} k_{2} k_{3} k_{4}}$ in Eq. (11) describe two-particle inter-site transitions $\left(k_{4}, k_{3}\right) \leftrightarrow$ $\left(k_{2}, k_{1}\right)$ of the renormalized fermions. For a given transition, $\varkappa$ is simply the number of virtual steps that have to be made by the original fermions. The steps are counted off from the configuration where two such fermions occupy neighboring sites, and each step is a transition by one of the fermions to a nearest site. In other words, the original fermions go first from sites $\left(k_{4}, k_{3}\right)$ to sites $(p, p+1)$ and then to $\left(k_{2}, k_{1}\right)$ (we assume for concreteness that $k_{3}>k_{4}$ and $k_{1}>k_{2}$ ); the value of $p$ is chosen so as to minimize the number of steps.

To make the meaning of $\varkappa$ even more intuitive we give examples of some $\varkappa=4$ transitions. For the initial and final states $(n, n+1)$ and $(n-2, n+3)$ one of the sequences of steps of the original fermions is $(n, n+1) \rightarrow(n, n+$ $2) \rightarrow(n-1, n+2) \rightarrow(n-1, n+3) \rightarrow(n-2, n+3)$, whereas for the initial and final states $(n, n+2)$ and $(n-1, n+3)$ one of the sequences is $(n, n+2) \rightarrow(n, n+1) \rightarrow(n-$ $1, n+1) \rightarrow(n-1, n+2) \rightarrow(n-1, n+3)$ [the energy denominators must be obtained directly from Eqs. (8), (12)].

It follows from the above argument that, for large $\varkappa$ and $\alpha \gg \alpha_{\mathrm{th}}$, the transition matrix element

$$
V_{k_{1} k_{2} k_{3} k_{4}} \sim K^{\varkappa} \quad \text { for } \quad \varkappa \gg 1 .
$$

The transitions of renormalized fermions are not limited to nearest neighbors. However, from Eq. (14), the amplitudes of transitions over many sites are small and rapidly decrease with the number of involved virtual steps. The hopping integral for the renormalized fermions is $V J \Delta$. 
In higher orders of the perturbation theory, the interaction (11) leads also to many-particle transitions. The overall transition amplitude is determined by the total number of involved virtual single-particle steps.

In order to localize many-particle excitations, one has to suppress combinational many-particle resonances keeping in mind that, for localization, the effective hopping integral must be smaller than the energy detuning of the initial and final on-site states. Because of the large number of possible resonances, we do not have an analytical proof of many-particle localization for our energy sequence (4). Instead we used numerical analysis, as described in the next section, which enabled us to demonstrate strong localization of stationary states in a chain of a limited size.

\section{B. The many-particle inverse participation ratio}

As mentioned earlier, a good indicator of strong localization, which applies to both single- and many-particle stationary states, is closeness of the IPR to one. In this section we present numerical data on the IPR obtained by diagonalizing the Hamiltonian (2) in the presence of several excitations. The Hamiltonian is a sparse matrix, which is separated into uncoupled blocks with different numbers of excitations. We focused on the block in the middle of the energy spectrum. For a given chain length $L$, it has $L / 2$ excitations and therefore the largest number of states. This is the worst case, in terms of localization. Chains with $L=10,12$, and 14 were studied. The results were similar. We present the data for $L=12$, in which case the total number of states is 924 .

The IPR as a function of the dimensionless parameter $\alpha$ of the on-site energy sequence (4) is shown in Fig. 4 The results refer to two values of the dimensionless ratio of the hopping integral to the interband distance $J / h$ and several values of the dimensionless parameter of the particle interaction $\Delta$.

We start the analysis with the region $\alpha \rightarrow 0$, where the on-site energies (4) alternate between $\pm h / 2$. For large $h / J$ the single-particle energies form bands of width $J^{2} / 2 h$. In the neglect of mixing of these bands, the manyparticle wave functions can be found using the Bethe ansatz. For $\Delta=0$ the many-particle energy spectrum consists of bands that are determined by the number of particles in each of the two single-particle bands. Because the states are of the plane-wave type, the IPR is large, with $\left\langle I_{6}\right\rangle \approx 78$ for $h / J=20$ and $L=12$, see Fig. 4

For $\alpha \rightarrow 0$ the IPR decreases with the increasing parameter of the particle interaction $\Delta$. This happens, because for large $\Delta \gg J / h$ (but $\Delta \ll h / J$ ) the energy bands at $\pm h / 2$ split into subbands depending on the number of particle pairs, triples, etc. on neighboring sites: for example, the energy of a pair on neighboring sites differs from the energy of a dissociated pair by $J \Delta$. Since the number of states in a subband is smaller than in the whole band, such splitting reduces the average IPR.

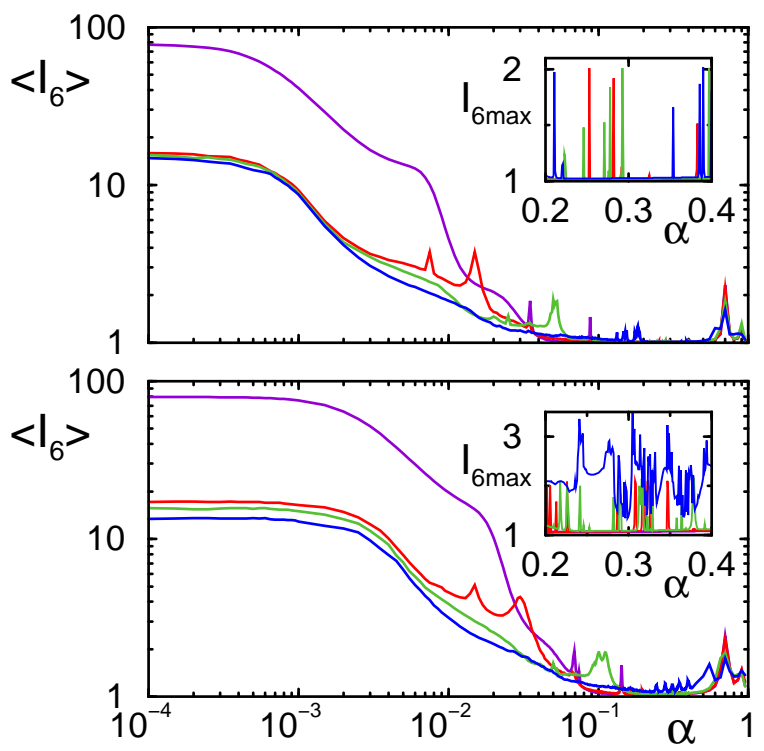

FIG. 4: (color) The IPR for 6 excitations on the first 12 sites of the chain (4). The reduced bandwidth of the energy spectrum is $h / J=20$ (top panel) and $h / J=10$ (lower panel). The purple, red, green, and blue curves refer to the coupling parameter $\Delta=0,0.3,1$, and 3 , respectively. The peaks of $\left\langle I_{6}\right\rangle$ for $\Delta=0$ are a single-particle boundary effect. The insets show the maximal $I_{6}$ for $\Delta \neq 0$. Sharp isolated peaks of $I_{6 \max }$ vs $\alpha$ result from the hybridization of resonating onsite many-particle states.

The decrease of the average IPR with increasing $\Delta$ for $\alpha \rightarrow 0$ and $\Delta \ll h / J$ is seen in Fig. 4 We note that the mere separation (by $h$ ) of the single-particle energies of neighboring states is not sufficient for strong localization. Inside each subband, the number of resonating states in a long chain is still very large. Localization requires that not only nearest neighbor single-particle energies, but also energies of remote sites be tuned away from each other. This should happen for sufficiently large values of the parameter $\alpha$ in Eq. (4).

This argument is confirmed by the data in Fig. 4. For given $\Delta$, the IPR decreases as a whole with increasing $\alpha$ in the region where the single-particle bands are well separated, $\alpha \lesssim 0.4$. This is the expected consequence of elimination of energy resonances.

In the region $0.2 \lesssim \alpha \lesssim 0.4$, except for narrow peaks, we have $I_{6 \max } \approx 1.09$ for $h / J=10$ and $I_{6 \max } \approx 1.02$ for $h / J=20$, for $\Delta \leq 1$. The values of $\left\langle I_{6}\right\rangle$ are even smaller, 1.04 and 1.01, respectively. This indicates that, in this parameter range, all states are strongly localized. For $\Delta=3$ and $h / J=20$ we also have $I_{6 \max } \approx 1.01$ away from the peaks; however, for $h / J=10$ the IPR becomes larger due to the many-particle resonances, which are discussed below (see also Appendix C).

A distinctive feature of the many-particle IPR as function of $\alpha$ is the onset of multiple resonant peaks, which can be seen in Fig. 4. They indicate that at least some 
of the stationary states are no longer confined to a single set of sites, with the number of sites equal to the number of particles. The peaks are due to hybridization of resonating on-site states. The hybridization occurs when the matrix elements of inter-site transitions in Eqs. (11), (12) exceed the energy difference of the states.

For $0.2 \lesssim \alpha \lesssim 0.4$, i.e., in the region of strong localization, and for $h / J=20$ and chosen $\Delta \leq 3$ we found that only two on-site states could become strongly hybridized in the section of the chain with $1 \leq n \leq 12$. Hybridization of a larger number of states was weaker. A consequence of hybridization of utmost two states is that $I_{6 \max } \lesssim 2$. For $h / J=10$ and $\Delta=3$ the interstate coupling (12) is stronger, and as a result three states can be strongly hybridized and a few more can be weakly admixed, leading to $I_{6 \max } \sim 3$ at resonant $\alpha$.

Because the interaction is two-particle, the strongest peaks of $I_{\max }$ come from resonances between on-site energies of two particles. They occur when the energy difference

$$
\delta \varepsilon=\left|\varepsilon_{k_{1}}+\varepsilon_{k_{2}}-\varepsilon_{k_{3}}-\varepsilon_{k_{4}}\right|
$$

is close to $M J \Delta$ with $M=0,1,2$. Strictly speaking, we should use exact single-particle energies $\varepsilon_{n}^{\prime}$ (9) instead of $\varepsilon_{n}$ in Eq. (15), but the difference between these energies is small, see Eq. (10), and it leads to a small shift of the positions of the resonances as functions of $\alpha$. An explanation of the positions and widths of the narrow peaks of $\left\langle I_{6}\right\rangle$ and $I_{6} \max$ seen in Fig. 4 is given in Appendix C.

\section{Broad-band two-particle resonances}

A special role in the problem of many-body localization is played by two-particle resonances that are not selective in $\alpha$, i.e., exist in a broad range of $\alpha$. For these broadband resonances, the total energy difference between the initial and final on-site states is small, $\delta \varepsilon \ll J$ even for small $J / h$. They emerge already in the second order in $J / h$, when only two single-particle steps to neighboring sites are required, $\varkappa=2$. The resonating on-site states are pairs $(n, n+1)$ and $(n-1, n+2)$, i.e.,

$$
\varepsilon_{n}+\varepsilon_{n+1} \approx \varepsilon_{n-1}+\varepsilon_{n+2} .
$$

If $n$ and $n+2$ are prime numbers, the energy difference $\delta \varepsilon=\left|\varepsilon_{n}+\varepsilon_{n+1}-\varepsilon_{n-1}-\varepsilon_{n+2}\right| \sim \alpha^{n-1} h$ is extremely small for large $n$.

More generally, the resonance (16) occurs for all $n=$ $6 k-1$ with integer $k$. In this case $\delta \varepsilon / h \propto \alpha^{\xi}$ with $\xi \geq 4$. Such $\delta \varepsilon$ is "anomalously small" for $\varkappa=2$. The hopping integral $J^{3} \Delta / h^{2}$ becomes larger than $\delta \varepsilon$ even when we are already deep in the single-particle localization region, $\alpha \gg \alpha_{\text {th }}$.

As a result of renormalization $\varepsilon_{n} \rightarrow \varepsilon_{n}^{\prime}$, the energy difference $\delta \varepsilon=\left|\varepsilon_{n}+\varepsilon_{n+1}-\varepsilon_{n-1}-\varepsilon_{n+2}\right|$ is changed. For $n=6 k-1$ to leading-order in $J / h$ the renormalization of $\delta \varepsilon$ does not exceed $\sim \alpha^{3}\left(J^{2} / h\right)$ or $\sim \alpha^{4}\left(J^{2} / h\right)$ for odd and even $k$, respectively (it may also be proportional to higher power of $\alpha$ ). The renormalized $\delta \varepsilon$ can still be much smaller than $J^{3} \Delta / h^{2}$, and then the broadband resonance persists. More many-particle broad-band resonances emerge for larger $\varkappa$.

For the section of the chain with sites $1 \leq n \leq 12$ the IPR is not much affected by the broad-band resonances, because even where $n$ and $n+2$ are prime numbers ( 5 and 7 ), they are not very large and the energy difference (16) is not exceedingly small.

The situation changes if we consider other sections of the chain (4) of the same length, $n_{0} \leq n \leq n_{0}+11$ with different $n_{0}$. Here we found that the resonances increase $\left\langle I_{6}\right\rangle$ up to 1.15 between the peaks, for $h / J=20, \Delta=1$, and for all $\alpha$ in the interval $0.2<\alpha<0.4$.

For a finite chain, the resonances can be eliminated order by order in $\varkappa$ by shifting the energies of the appropriate qubits. Simple systematic modifications of the energies that work for $\varkappa \leq 5$ are discussed in the next section, see Eqs. (19), (22). Both modifications bring the IPR back to smaller values. For example, in all sections of the chain that we studied the made $\left\langle I_{6}\right\rangle$ and $I_{6} \max$ equal to $\approx 1.01$ and $\approx 1.02$, respectively, which are the values we had for the section $1 \leq n \leq 12$. This indicates that the localization of stationary states for the modified energy sequences is very strong.

\section{LIFETIME OF STRONGLY LOCALIZED STATES}

The problem of strong localization can be viewed also from a different perspective. In the context of quantum computing, it suggests a more appropriate formulation then the one based on the analysis of stationary states. It is also relevant for condensed-matter systems at nonzero temperatures.

First we note that excitations in quantum computers and in condensed-matter systems have a finite coherence time $t_{\mathrm{coh}}$. For QC's, this time has to be compared with the duration of a single- or two-qubit operation and measurement. The duration of a two-qubit operation is of order of the time it takes to resonantly transfer an excitation between the qubits, which is $\sim J^{-1}$. A single-qubit operation is often faster; however, the measurement can sometimes be slower. In most proposed realizations of a QC the coherence time exceeds the gate operation time by a factor $\lesssim 10^{5}$.

We define the localization lifetime $t_{\text {loc }}$ as the time it takes for excitations to leave occupied sites. Localization of excitations is only relevant on times $\sim t_{\text {coh }}$. Then to have strong localization it suffices that $t_{\mathrm{loc}} \gtrsim t_{\mathrm{coh}}$. It follows from the estimate for $t_{\mathrm{coh}}$ that the latter condition is met if

$$
t_{\mathrm{loc}} \gtrsim 10^{5} J^{-1}
$$

The condition (17) must be satisfied for all on-site manyexcitation states. It is this condition that imposes a con- 
straint on the form of the energy sequence $\varepsilon_{n}$ in an infinite many-particle system.

The time $t_{\text {loc }}$ is determined by hopping between resonant on-site states. It occurs through virtual transitions via nonresonant sites. For a two-particle resonant transition, the minimal number of the needed virtual steps is given by the parameter $\varkappa$ (13). Then from Eqs. (11), (14) the hopping integral for a resonant transition $\left(k_{4}, k_{3}\right) \leftrightarrow\left(k_{2}, k_{1}\right)$ is

$$
J \Delta V_{k_{1} k_{2} k_{3} k_{4}} \sim J \Delta K^{\varkappa}
$$

for $\varkappa \gg 1$. Here, $K$ is defined by Eq. (6), $K \approx J / 2 \alpha h$, and $K \ll 1$ in the region $\alpha / \alpha_{\text {th }} \gg 1$.

The localization lifetime $t_{\text {loc }}$ is determined by the reciprocal maximal hopping integral for resonating manyparticle states. In the case of an energy sequence of the type (4), up to a fairly high number of virtual steps $(\leq 5)$, of interest are resonances between two-particle on-site states. This applies to systems with an arbitrary number of particles; only those transitions matter in which up to two particles change sites. Indeed, transitions where three particles change sites emerge in the second order in the two-particle Hamiltonian (11), (12), and simple counting shows that they involve already at least 5 virtual steps.

For resonant two-particle transitions $t_{\text {loc }} \sim$ $\min \left[J \Delta K^{\varkappa}\right]^{-1}$. Therefore it strongly depends on the minimal value of $\varkappa$ for all pairs of resonating initial and final on-site states, $\varkappa=\varkappa_{\min }$. To have $t_{\text {loc }} J$ that exceeds a given value, we must have an appropriate $\varkappa_{\text {min }}$. This means that we should eliminate resonances between all states connected by $\varkappa<\varkappa_{\text {min }}$ virtual transitions.

A two-particle transition with odd $\varkappa=1,3,5, \ldots$ involves a change of the total number of occupied sites with even $n$ (and by the same token, with odd $n$, too). Therefore, for the sequence (4) with $\alpha \ll 1$, the energy change in such a transition is $\delta \varepsilon \sim h$. In this paper we consider the case of weak to moderately strong coupling, when $\Delta \lesssim 1$. Then $\delta \varepsilon$ significantly exceeds the change of the interaction energy $J \Delta, 2 J \Delta$ for $h \ll J$. As a result, resonant two-particle transitions may occur only for even $\varkappa$.

We will modify the sequence (4) to eliminate resonances with $\varkappa=2$ and $\varkappa=4$. In these cases $\varkappa_{\text {min }}=4$ and 6 , respectively, leading to the localization time $t_{\text {loc }} \sim$ $J^{-1} K^{-4}$ and $>J^{-1} K^{-6}$.

\section{A. Eliminating second order many-particle resonances}

The potentially resonant transitions with $\varkappa=2$ are

$$
\begin{aligned}
& (n, n+1) \leftrightarrow(n, n+1 \pm 2),(n-2, n+1) \\
& (n, n+1) \leftrightarrow(n-1, n+2) .
\end{aligned}
$$

In the transitions listed in the first line of this equation, one of the particles in the pair moves by two sites in one or the other direction, whereas for the transition shown on the second line both particles move by one site.

The number of occupied nearest sites in the transitions (18) can change by one or remain unchanged. Therefore the maximal change of the interaction energy is $J \Delta$. Second-order resonances will be eliminated if the detuning of the on-site energy differences $\delta \varepsilon$ for the transitions (18) is

$$
\delta \varepsilon>J \Delta .
$$

This means that we need a zero-energy gap of an appropriate width in $\delta \varepsilon$.

We note that this is a sufficient, not the necessary condition. In principle, it would suffice to have narrow gaps at $\delta \varepsilon=0$ and $J \Delta$. These gaps should just be broader than the tunneling matrix element and than the energy shifts due to occupation of next nearest neighbors. For a specific finite-length section of a chain this may be more practical. However, here we are interested in an infinite chain, and we want to demonstrate that even for such a chain all resonances with $\varkappa<4$ can be eliminated.

To create the zero-energy gap, sequence (44) has to be modified. The modification has to eliminate, in the first place, the "anomalous" broad-band resonances for transitions $(n, n+1) \leftrightarrow(n-1, n+2)$ with $n=6 k-1$ discussed before. This will, of course, also eliminate resonances where $n$ and $n+2$ are prime numbers. A simple and sufficient modification is a constant shift of $\varepsilon_{n}$ for each 6 th site,

$$
\varepsilon_{n}^{\mathrm{md}}=\varepsilon_{n}+(h / 2) \alpha^{\prime} \quad \text { for } \quad n=6 k,
$$

while $\varepsilon_{n}^{\mathrm{md}}=\varepsilon_{n}$ for $n \neq 6 k$.

For the modified sequence (19), the gap in the onsite energies for the 2nd-order transitions (18) is $\delta \varepsilon \sim$ $\alpha^{2} h, \alpha^{\prime} h / 2$ to leading order in $\alpha$. A more accurate estimate is $\min \delta \varepsilon / h \approx \alpha^{2}-\alpha^{3}, \alpha^{\prime} / 2$. We assume that $\alpha^{2} \lesssim \alpha^{\prime} \ll \alpha$, in which case no new resonances are created for the transitions (18) as a result of the modification (19).

It follows from the above estimate that, for an infinite chain and an arbitrary number of particles, all resonant transitions with $\varkappa<4$ will be eliminated provided $J \Delta / h<\alpha^{2}-\alpha^{3}, \alpha^{\prime} / 2$, . Then the localization time $t_{\text {loc }} \sim 10^{5} J^{-1}$ already for $h / J=30, \alpha=0.3$, $\alpha^{\prime} \approx 0.1-0.2$, and $\Delta \lesssim 1$.

The effect of the on-site energy modification (19) on localization of a many-particle state is seen from Fig. 5 This figure shows the dynamics of the system prepared initially in the state $|\Phi(416,419,420,422,423,424)\rangle$. In the case of the original sequence (4), this state strongly hybridizes with the state $|\Phi(416,418,421,422,423,424)\rangle$ for all $\alpha$ of physical interest, $\alpha<0.4$. This happens because the difference of on-site energies $\varepsilon_{419}+\varepsilon_{420}$ and $\varepsilon_{418}+\varepsilon_{421}$ is $\delta \varepsilon \sim \alpha^{418} h$. The hybridization results in oscillations of the amplitude of the state, as seen from Fig. 5] For the modified sequence (19) the resonance is eliminated, and the amplitude remains constant over 


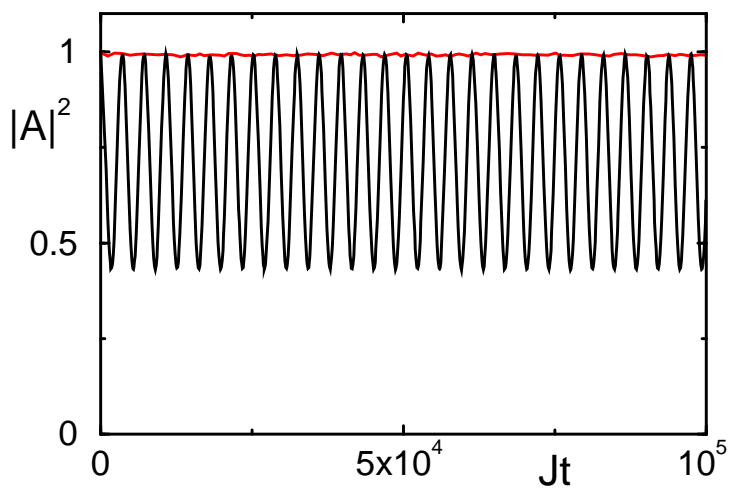

FIG. 5: (color online) Time evolution of the squared amplitude $|A|^{2}$ of the on-site state $|\Phi(416,419,420,422,423,424)\rangle$ in a 12-site section of the chain between $n=415$ and $n=426$. The oscillating line refers to the original sequence (4) with $\alpha=0.25$. The nearly constant line refers to the modified sequence (19) with $\alpha=0.25, \alpha^{\prime}=0.22$. In both cases $h / J=20$ and $\Delta=1$.

a time $>10^{6} \mathrm{~J}^{-1}$. This illustrates the onset of strong localization. We note that the localization time of this particular state turns out to be longer for the modified sequence (19) than the worst-case estimate given above.

\section{B. Eliminating 4th order resonances}

The localization time is further dramatically increased if $\varkappa=4$ resonances are eliminated. The potentially resonant 4 th order transitions are

$$
\begin{aligned}
(n, n+1) & \leftrightarrow(n-2, n+3), \\
(n, n+1) & \leftrightarrow(n+2, n+3), \\
(n, n+3) & \leftrightarrow(n-1, n+2), \\
(n, n+3) & \leftrightarrow(n-2, n+1),
\end{aligned}
$$

and

$$
\begin{aligned}
(n, n+1) & \leftrightarrow(n-1, n+4), \\
(n, n+1) & \leftrightarrow(n-3, n+2), \\
(n, n+1) & \leftrightarrow(n, n+1 \pm 4),(n \pm 4, n+1)
\end{aligned}
$$

In the last line of Eq. (21) we list transitions where one of the particles in the pair moves by 4 sites, whereas in all other transitions both particles move away from their sites.

For the modified sequence (19), the minimal energy change in the transitions (20), (21) is $\min \delta \varepsilon \sim \alpha^{3} h$, to leading order in $\alpha$, provided $\alpha^{\prime} \gg \alpha^{3}$. The value of $\alpha^{\prime}$ has to be in such a range that the modification (19) does not lead to extra resonances between the on-site energies for the states (20) and (21). The "dangerous" combinations in $\delta \varepsilon / h$ are $\left|\alpha-\alpha^{\prime} / 2\right|,\left|2 \alpha-\alpha^{\prime} / 2\right|,\left|\alpha^{2}-\alpha^{\prime} / 2\right|,\left|2 \alpha^{2}-\alpha^{\prime} / 2\right|$, to leading order in $\alpha$. We will choose $\alpha, \alpha^{\prime}$ so that all of them exceed $\min \delta \varepsilon / h \approx \alpha^{3}$.

Fig. [6] shows how the modification (19) leads to a zeroenergy gap in $\delta \varepsilon$. We plot $\delta \varepsilon_{n}^{\mathrm{md}}$ for all transitions (20), (21) with one of the particles on site $n$ (with $n>2$ ). Therefore we show all potentially resonant transitions with $\varkappa \leq 5$.
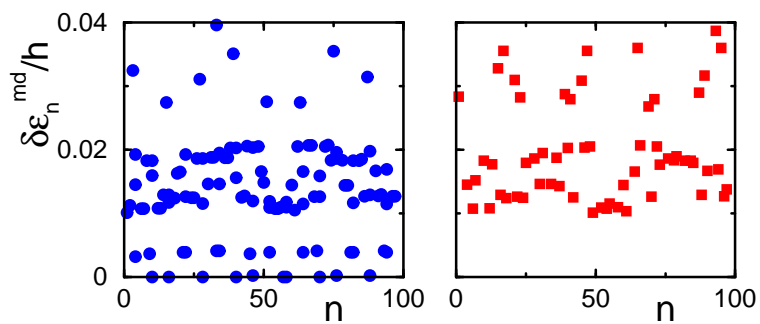

FIG. 6: (color online) The low-energy part of the two-particle energy differences $\delta \varepsilon_{n} / h$ (15) for all transitions with $\varkappa \leq 5$ in which one of the involved particles is on the $n$th site $(n>2)$. The data refer to $\alpha=0.25$. The left panel corresponds to the sequence (4). The right panel refers to the modified sequence (19) with $\alpha^{\prime}=0.22$ and shows the zero-energy gap in $\delta \varepsilon$.

The left panel in Fig. 6 shows that, for the initial sequence (4), there is practically no gap in the values of $\delta \varepsilon$ at low energies. The right panel demonstrates that the correction (19) leads to a zero-frequency gap. The gap depends on the values of $\alpha$ and $\alpha^{\prime}$. For the specific parameter values in Fig. [6] we have $\delta \varepsilon / h \geq 0.01$.

We have checked numerically that the gap persists for a much longer chain than shown in Fig. [6 with $n$ from 2 to 10,000 . This is more than enough to prove that the results apply to an infinite chain. Indeed, in $\varepsilon_{n}^{\mathrm{md}}$ the terms $\propto \alpha^{q}$ with different $q$ are repeated periodically with period $2(q+1)$. Therefore a sequence of terms with $\alpha^{q}$ for $q \leq 6$ is repeated periodically with period equal to twice the least common multiple of all $q+1 \leq 7$, which is $2 \times(3 \times 4 \times 5 \times 7)=840$ (cf. Appendix A). For $\alpha=0.25$ we have $\alpha^{6} \approx 2 \times 10^{-4}$. The contribution to $\varepsilon_{n}^{\mathrm{md}} / h$ of all terms of higher degree in $\alpha$ is then $\lesssim 2 \times 10^{-4}$. This means that, to accuracy better than $2 \times 10^{-4}$, the results on the gap for an infinite chain will coincide with the results for a chain of 840 sites.

It follows from the discussion above that, for $2 J \Delta \lesssim$ $0.01 h$, all particles will remain localized on their sites for the time $t_{\text {loc }} \sim(J \Delta)^{-1} K^{-6} / \alpha$ [we have taken into account here that the hopping integral for transitions with $\varkappa=6$ is limited by $\sim J \Delta(J / 2 h)^{6} \alpha^{-5}$ rather than $J \Delta(J / 2 h)^{6} \alpha^{-6}$, as would be expected from the asymptotic expression (14)]. For $h / J=50$ and $\alpha=0.25$ this gives an extremely long localization time, $t_{\mathrm{loc}} J \gtrsim 10^{10}$. However, this estimate requires that the coupling be weak, $\Delta \lesssim 0.25$ for the used parameter values. 


\section{Extension to stronger coupling}

The previous result can be easily extended to larger $\Delta$ without increasing $h$. To do this we note that the change of the interaction energy in transitions (20) is actually limited to $J \Delta$ rather than $2 J \Delta$, which is the case for the transitions (21). Therefore the gap for the transitions (21) should be twice as large as for the transitions (20). Two first transitions (21) have a gap $\gtrsim \alpha^{2} h$ to leading order in $\alpha$, but for the last one $\min \delta \varepsilon=\alpha^{3} h$. This latter gap may be increased by choosing a somewhat different modification of the on-site energy spectrum. In contrast to Eq. (19), we will shift the energy of each 3rd site,

$$
\tilde{\varepsilon}_{n}^{\mathrm{md}}=\varepsilon_{n}-(h / 4) \beta\left[1+3(-1)^{k}\right] \quad \text { for } \quad n=3 k,
$$

while $\tilde{\varepsilon}_{n}^{\mathrm{md}}=\varepsilon_{n}$ for $n \neq 3 k$.

Eq. (22) corresponds to shifting $\varepsilon_{3 k}$ up by $\beta h / 2$ or down by $\beta h$ depending on whether $k$ is odd or even, respectively. The parameter $\beta$ should be much larger than $\alpha^{3}$, to open a gap $\sim \min \left(\alpha^{2} h, \beta h / 2\right)$ in $\delta \tilde{\varepsilon}_{n}^{m d}$ for the transitions (21). At the same time, it should be chosen so as to avoid creating new resonances, similar to the modification (19).

It is straightforward to show that the modification (22) leads to a zero-energy gap in $\delta \varepsilon$. For $\alpha=0.25, \beta=0.1725$ the gap exceeds $0.01 h$ for the transitions (18), 20) with energy change $\lesssim J \Delta$, whereas for the transitions 21) with energy change up to $2 J \Delta$ it exceeds $0.02 h$. This indicates that the results on the localization time $t_{\text {loc }} J \gtrsim$ $10^{10}$ for $h / J=50$ now apply for $\Delta \lesssim 0.5$.

As in the previous section, the extremely large localization time characterizes an infinite chain and an arbitrary number of interacting particles. We note that both modifications of the original energy sequence, Eqs. (19) and (22), are obtained analytically, by finding the leadingorder terms in the energy differences for the transitions (18), (20), and (21). The specific values of the parameters $\alpha, \alpha^{\prime}$, and $\beta$ are used just to illustrate the order of magnitude of the localization time.

\section{STABILITY WITH RESPECT TO ERRORS IN ON-SITE ENERGIES}

In a real system, it will be impossible to implement sequence of on-site energies (4) precisely. This is because these energies contain high powers of the small parameter $\alpha$, while the precision to which they can be set and/or measured is limited. Therefore it is necessary to study localization in the presence of errors in $\varepsilon_{n}$ and to find how large these errors can be before they cause delocalization.

We will address this problem by looking at the gap in the energy differences $\delta \varepsilon$ in the presence of errors in $\varepsilon_{n}^{\mathrm{md}}$ (19). As long as this gap remains larger than $2 J \Delta$ for all resonant transitions with $\varkappa \leq 5$, the localization lifetime $t_{\text {loc }}$ will remain large. The analysis can be immediately extended to the modified sequence (22) as well.
The effect of errors on the gap can be modelled by adding a random term to on-site energies, i.e., replacing $\varepsilon_{n}^{\mathrm{md}}$ with

$$
\varepsilon_{n}^{\mathrm{err}}=\varepsilon_{n}^{\mathrm{md}}+\frac{1}{2} D h r_{n} .
$$

Here, $r_{n}$ are random numbers uniformly distributed in the interval $(-1,1)$, and $D$ characterizes the error amplitude. It should be compared with $\alpha^{s}$ with different exponents $s \geq 1$. When $D \sim \alpha^{s}$ it means that the energies $\varepsilon_{n}$ are well controlled up to terms $\sim \alpha^{s-1}$, to leading order in $\alpha$.

From the above arguments it follows that, for $\alpha \gg$ $\alpha^{\prime} \gtrsim \alpha^{2}$ the gap should remain unchanged if $D \ll \alpha^{4}$. This is because, for the modified energies $\varepsilon_{n}^{\mathrm{md}}$, the terms $\sim \alpha^{4}$ drop out from the energy differences that we discuss. For $D \sim \alpha^{4}$ the gap should be somewhat reduced. For $D \sim \alpha^{3}$ it should become significantly smaller than for $D=0$, and it should ultimately disappear with increasing $D$.

Numerical results on the gap $\delta \varepsilon$ as a function of $\log D$ are shown in Fig. 7. The gap is calculated for two-particle transitions with $\varkappa \leq 5$, as in Fig. [6] In the lower panel the gap is scaled by its value in the absence of errors,

$$
R=\min _{n} \delta \varepsilon_{n}^{\mathrm{err}} / \min _{n} \delta \varepsilon_{n}^{\mathrm{md}} .
$$

The data refer to the same $\alpha, \alpha^{\prime}$ as in Fig. 6. They are in full agreement with the above estimate.
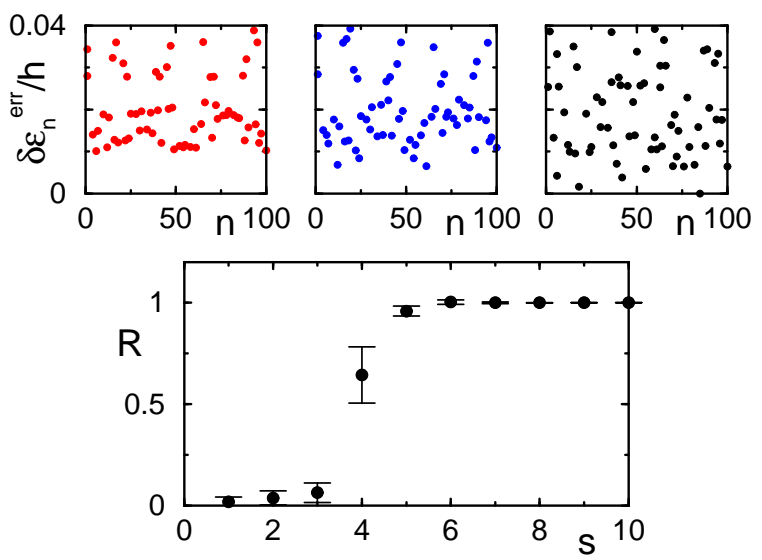

FIG. 7: (color online) Upper panels: all energy differences $\delta \varepsilon_{n}^{\text {err }} / h=\left|\varepsilon_{n}^{\text {err }}+\varepsilon_{n_{1}}^{\text {err }}-\varepsilon_{n_{2}}^{\text {err }}-\varepsilon_{n_{3}}^{\text {err }}\right| / h$ for the transitions (18), (20), (21) that correspond to the number of intermediate steps $\varkappa \leq 5$. The data refer to $\alpha=0.25, \alpha^{\prime}=0.22$, and to a specific realization of the random numbers $r_{n}$ in Eq. (23). The boxes from left to right correspond to the values of the noise intensity $D=\alpha^{s}$ in Eq. (23) with $s=5,4$, and 3 . Lower panel: the scaled minimal gap $R$ (24) as a function of the $\operatorname{logarithm} s=\ln D / \ln \alpha$ averaged over 10 realizations of noise. Error bars show the standard deviation of $R$.

The results of Fig. [7demonstrate that the localization persists even for relatively large errors in the on-site energies. At least for the chosen $\alpha$ and $\alpha^{\prime}$, errors in $\varepsilon_{n}$ up 
to $\sim 0.4 \%$ (when $D=\alpha^{4}$ ) lead to a change in the width of the energy gap by $\sim 50 \%$.

The observed dependence on the noise strength suggests that, in the presence of noise, sequence (4), (19) can be cut so that the terms $\propto \alpha^{s}$ with $s>s_{\text {cutoff }}$ are disregarded. The value of $s_{\text {cutoff }}$ depends on the noise, $s_{\text {cutoff }}=\ln D / \ln \alpha$. As a result of the cutoff, the energies $\varepsilon_{n}$ become polynomials in $\alpha$ of power $\leq s_{\text {cutoff }}$. From Eq. (4), these polynomials are periodic in $n$, with the period determined by twice the least common multiple of $\left(2,3, \ldots, s_{\text {cutoff }}+1\right)$. For example, for $s_{\text {cutoff }}=5$ the period in $n$ is 120 (see also Appendix A). For such a long period and short-range hopping, excitations will stay on their sites for a long time compared to $J^{-1}$.

\section{ALTERNATIVE ENERGY SEQUENCES}

Neither the original sequence of on-site energies (4) nor its modified versions (19), (22) were optimized to maximize the IPR or the localization lifetime. Therefore it is important to compare them with other sufficiently simple sequences. This will be done in this section for two natural choices of $\varepsilon_{n}$.

\section{A. Period doubling cascade}

A simple way to move sites with close energies far away from each other is to make the energies form a "period doubling cascade" (PDC). It can be described by a oneparameter energy sequence; in what follows $h$ is the energy scale and $\alpha$ is the parameter, as in Eq. (4).

In the PDC, the on-site energies are first split into two subbands, with nearest neighbors being in different subbands, but next nearest neighbors being in the same subband. The subbands differ in energy by $\alpha^{0} h$, to leading order in $\alpha$ [this is also the case in Eq. (4)]. Each of the subbands is then further split into two subbands of 4th neighbors. The leading term in the energy difference of these subbands is $\alpha^{1} h$. Each subband is then split again into two subbands of 8 th neighbors. The leading term in their energy difference is $\alpha^{2} h$. This period-doubling process is then continued indefinitely, for an infinite chain.

The expression that describes the on-site energy sequence for the PDC, $\varepsilon_{n}^{\mathrm{PDC}}$, can be conveniently written in terms of the coefficients $j_{k}(n)=0,1$ of the expansions of site numbers $n$ in base two,

$$
n=\sum_{k=0}^{M(n)-1} j_{k}(n) 2^{k} .
$$

Here, $M(n)=1+\left\lfloor\log _{2} n\right\rfloor$ is the number of integer digits of $n$ in base 2 .

We set

$$
\varepsilon_{n}^{\mathrm{PDC}}=\frac{1}{2} h\left[(-1)^{n}+\sum_{k=1}^{M(n)-1}(-1)^{j_{k}(n)} \alpha^{k}\right] .
$$

The energies $\varepsilon_{n}^{\mathrm{PDC}}$ are shown in Fig. 8 It is instructive to compare this figure with Fig. 1 for the energies (4). The overall band structure is similar, but the energy distribution is much more regular for the PDC. For example, it is seen from the panels for 2000 sites that the minibands for the PDC sequence have approximately equal numbers of states, in contrast to Fig. 11. However, as we show later, the symmetry of the PDC is actually bad from the viewpoint of many-particle localization.
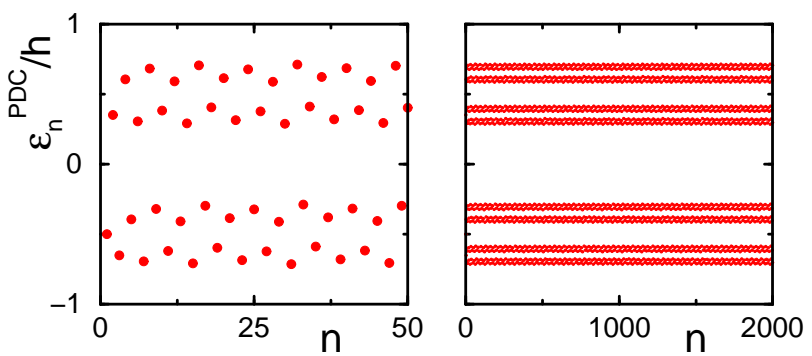

FIG. 8: (color online) The on-site energies $\varepsilon_{n}^{\mathrm{PDC}} / h$ (25) for $\alpha=0.3$. The left panel shows the energies for the sites $n=1,2, \ldots, 50$. States with close on-site energies are spatially separated. The right panel shows $\varepsilon_{n}^{\mathrm{PDC}} / h$ for a much longer array, $n=1, \ldots, 2000$. The energy spectrum displays a multisubband structure. Because of the symmetry of sequence [25], the number of points in each subband is approximately the same.

Spatial separation of sites with close energies in the PDC leads to effective localization of single-particle stationary states. As in the case of sequence (4), the values of $\left\langle I_{1}\right\rangle-1$ are $\sim 3 \times 10^{-3}$ for $h / J=20$ near the minimum of $\left\langle I_{1}\right\rangle$ over $\alpha$, in agreement with the estimate (7). The minimum of $\left\langle I_{1}\right\rangle$ is located at $\alpha \approx 0.1$.

The situation is different for many-particle localization. Here the high symmetry of the PDC leads to multiple many-particle resonances. For example, two-particle states $(n, n+1)$ and $(n-1, n+2)$, which are coupled in second order in $J / h$, have equal energies whenever $n$ is odd. The states $(n, n+1)$ and $(n-2, n+3)$, that are coupled in the fourth order in $J / h$, have the same energies when $n=7+4 k$ with integer $k$. We note that there were no exactly degenerate states for sequence (4), and the portion of two-particle states with close energies was much smaller. Therefore it is more complicated to find a correction to sequence (25) that would eliminate many-particle resonances. As a result, unexpectedly, this symmetric sequence is less convenient from the point of view of strong localization.

\section{B. Random on-site energies}

The case opposite to the highly symmetric sequence (25) is when the on-site energies $\varepsilon_{n}$ are completely random. It is well-known that such randomness leads to single-particle localization of stationary states in a $1 \mathrm{D}$ 
chain. However, it does not lead to strong on-site localization of all states, because there is always a nonzero probability to have neighboring sites with energies that differ by less than $J$ and therefore are hybridized. As explained in the Introduction, hybridization is even more likely to happen in the case of many-particle states, because it is more likely to have neighboring nearly resonant sites.

A simple random sequence of on-site energies has the form

$$
\varepsilon_{n}^{\mathrm{r}}=W r_{n}^{\prime}
$$

where $r_{n}^{\prime}$ with different $n$ are independent random numbers uniformly distributed in the interval $(0,1)$, and $W$ is the bandwidth. The results on the inverse participation ratio for sequence (26) are shown in Fig. 9 .
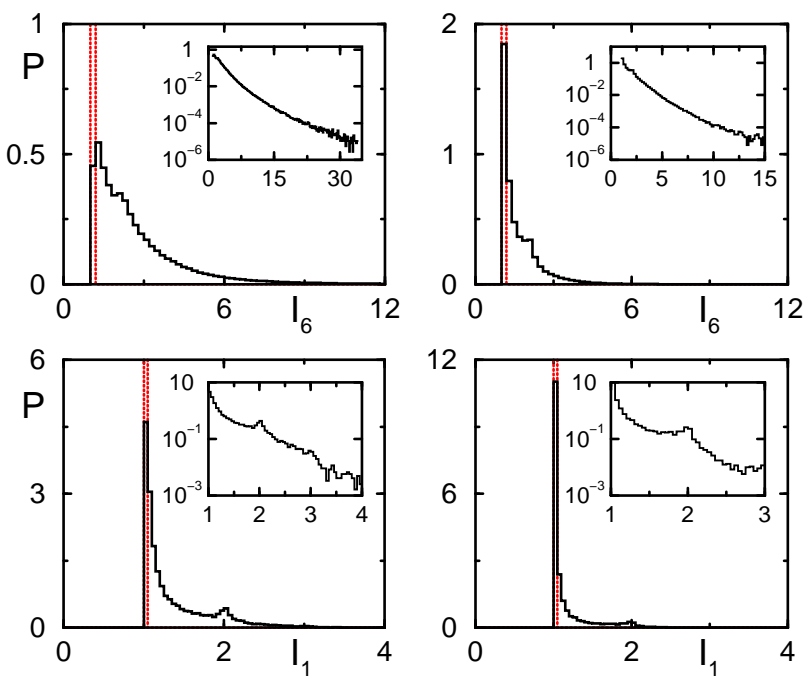

FIG. 9: (color online) The normalized distributions of the IPR $P(I)$ for the random energy sequence (26). The upper and lower panels refer to the many- and single-particle IPR's: 6 excitations with $\Delta=1$ in the chain of length $L=12$ and one excitation in the chain of $L=300$, with 2000 and 80 realizations, respectively. The left and right panels refer to the overall bandwidth of the on-site energy spectrum $W / J=13$ and 26. The narrow columns at $I=1$ showed with dotted lines refer to sequence (4), with $h / J=10$ and 20 for the left and right panels, respectively. The values of $\alpha$ in Eq. (4) were chosen so as to have the same bandwidths of on-site energies as the corresponding random sequences: in the lower panels $\alpha \approx(W-h) / W \approx 0.231$ (as in an infinite chain), whereas in the upper panels $\alpha=0.274$ to allow for a comparatively small number of sites. The insets show $\log P$ vs. $I$.

Localization of stationary states for the random sequence (26) can be characterized by the probability distribution of the IPR $P(I)$. This distribution was obtained by numerically diagonalizing the Hamiltonian (2) for different realizations of the on-site energies (26).

As seen from Fig. 9] both single- and many-particle IPR distributions display peaks near $I=1$ for random on-site energies. This indicates that, for the wide energy bandwidths used in the calculations, most of the stationary states of the system are strongly localized. However, the distributions are broad and slowly decay on the tails. This means that many on-site states are strongly hybridized, that is the stationary wave functions spread over several sites. This is a consequence of multiple resonances. The insets in Fig. 9] show that, at least for not too large $I$, the tails of $P(I)$ are non-exponential.

The typical distribution width decreases with the increasing bandwidth $W$ of on-site energies. As expected, the distributions of many-particle IPR's (the upper panels in Fig. 9) are much broader and their peaks near $I=1$ are much smaller than for the single-particle IPR's (the lower panels).

The IPR distributions for the random sequence (26) differ dramatically from the distributions for the regular sequence (4). The latter are narrow and concentrate in a small region of $I$ close to $I=1$, for the chosen bandwidths, both for the single- and many-particle states, cf. Figs. 3. 4. This is another indication of strong singleand many-particle localization for sequence (4).

\section{CONCLUSIONS}

In this paper we have explored two aspects of the problem of strong many-particle localization. One is localization of stationary states. For one-particle states, it has been studied analytically. We found that the wave functions decay quasi-exponentially and obtained the bounds on the decay length. The numerical results on strong localization are in agreement with the theory.

For many-particle stationary states, the localization has been analyzed numerically. Such analysis is unavoidably limited to small chains. For a 12-site chain we have found that, for the on-site energy sequence (19) with the ratio of the single-site energy bandwidth to the hopping integral $\approx 28\left[h / J=20, \alpha=0.25, \alpha^{\prime}=0.22\right.$ in Eq. (19)], the inverse participation ratio differs from its value for the case of fully localized states by $<2 \%$. This deviation is due to a small nonresonant admixture of the wave functions of neighboring sites.

A different approach is based on studying the lifetime of on-site states. It is sufficient to have a localization lifetime $t_{\text {loc }}$ that exceeds the coherence time of the excitations. We have shown that such $t_{\text {loc }}$ can be achieved in a chain of an arbitrary length and with an arbitrary number of excitations. For the explicit construction of on-site energies (19), resonant transitions that lead to delocalization require at least 4 virtual nonresonant steps, and for comparatively weak particle-particle coupling even 6 virtual transitions. Even in the 4-step case this leads to the ratio of the delocalization rate to the inter-site hopping integral $\sim 10^{-5}$ already for the decay length of the amplitude of virtual transitions $\sim 0.35$, or for $K \sim 0.06$.

The results on localization can be tested with artificial condensed-matter structures, as it was done in the studies of the effects of quasiperiodicity [20] (see also 
Refs. 18, 19). A discussion of experimental implementations is beyond the scope of this paper. Good examples are proposed models of a quantum computer; we note, however, that studying localization does not require operations on qubits, and therefore does not require a fully operational quantum computer.

In terms of quantum computing, an advantageous feature of sequence (4) and its modification (19) is that one radiation frequency can be used to resonantly excite different qubits. This can be achieved by selectively tuning them to this frequency without bringing neighboring qubits in resonance with each other, or by sweeping the frequency of the targeted qubit through the radiation frequency and having a Landau-Zener-type interstate transition. A two-qubit gate can be conveniently done by selectively tuning neighboring qubits in resonance with each other and having a Landau-Zener type excitation swap 16].

In many cases localization is also a prerequisite for a projective measurement. This happens when the measured quantity is the probability for each qubit to be in the excited state. Often a measurement is much slower than the time $J^{-1}$ of resonant hopping to a nearest site; then $t_{\text {loc }}$ should exceed the measurement time. In our approach, localization does not require refocusing [4], which is not always easy to implement and which is sometimes incompatible with slow measurement.

Our energy sequence does not lead to delocalization of stationary states due to indirect resonant $n \leftrightarrow n+2$ transitions. Such transitions undermine the approach to quantum computing with "always-on qubit interaction" proposed in Ref. 12, where next nearest neighbors are tuned in resonance. In terms of the localization lifetime, the width of the energy distribution (19) is orders of magnitude smaller than the width that would give the same $t_{\text {loc }}$ in the approach [12]. There the localization lifetime would be $\sim h / J^{2}$, where $h$ is the energy difference between nearest neighbors. This difference must exceed $J$ by a factor $\sim 10^{5}$ in order to have the same localization lifetime as in our approach. Compared to Ref. [11], a potential advantage of our approach is that the interaction does not have to be ever turned off, and no multi-qubit encoding is necessary for operating a QC.

The presented scheme can be extended to systems with long-range coupling. This is another distinction from the approaches 11, 12] that substantially rely on nearest neighbor coupling. For several proposed QC's the interqubit coupling is dipolar for a few near neighbors and becomes quadrupolar or falls down even faster for remote neighbors [5, 6, 9]. Long-range interaction makes transitions over several sites more probable. We leave detailed analysis for a separate paper. Here we note that, for sequence (22) and for single-particle transitions, not only hopping over 2 or 4 sites is not resonant and does not lead to delocalization, but even hopping over 6,8 , or 10 sites is nonresonant as well. For all these transitions, the energy difference is at least $\sim \alpha^{2} h$ or $\sim \beta h / 4$ [for sites separated by an odd number of positions, the energy difference is always large, $\sim h]$.

Our results provide proof of principle of strong on-site localization of all states, independent of the system size and the number of particles. We have not addressed the question of optimization of the energy sequence, so that maximal localization lifetime could be obtained for a minimal bandwidth of on-site energies. For a finite-length chain the optimization problem can be approached using Eq. (4) as an initial approximation and adjusting energies of several specific sites.

In conclusion, we have proposed a sequence of on-site energies (4) and its modifications (19), (22) that result in on-site localization of all single- and many-particle states of interacting spins or fermions in an infinite chain. The sequence (4) has low symmetry, which allows eliminating resonances between the states to a high order in the hopping integral. In turn, this leads to a long lifetime of localized many-particle states. When second-order resonances are eliminated, the lifetime exceeds the reciprocal hopping integral by 5 orders of magnitude provided the bandwidth of on-site energies is larger than the intersite hopping integral by a factor of $\sim 40$. We show that it can be further significantly increased by eliminating fourth-order resonances. The proposed energy sequence is stable with respect to errors. The results apply to scalable quantum computers with perpetually coupled qubits. They show that, by tuning qubit energies, excitations can be prevented from delocalizing between gate operations.

\section{Acknowledgments}

We are grateful to D.A. Lidar, L.P. Pryadko, and M.E. Raikh for helpful discussions. This work was partly supported by the Institute for Quantum Sciences at Michigan State University and by the NSF through grant No. ITR-0085922.

\section{APPENDIX A: EXPONENTIAL DECAY OF THE TRANSITION AMPLITUDE}

In this Appendix we give a rigorous proof of the quasiexponential decay of the amplitude $K_{n}(m)$ (5) of the transition from site $n$ to site $n+m$ and establish bounds on the decay length. We show that, for sequence (4), in the limit of small $\alpha$ and for $m \rightarrow \infty$

$$
\alpha^{-\nu_{L} m} \leq K_{n}(m)(2 h / J)^{m} \leq \alpha^{-\nu_{U} m} .
$$

We find that $\nu_{L} \geq 0.89$ and $\nu_{U} \leq 1.19$.

In order to simplify notations we introduce dimensionless energies $\varepsilon_{n}(\alpha)=2 \varepsilon_{n} / h$. From Eq. (4)

$$
\varepsilon_{n}(\alpha)=(-1)^{n}-\sum_{k=2}^{n+1}(-1)^{\lfloor n / k\rfloor} \alpha^{k-1} .
$$


We also set $J / 2 h=1$. Then $K_{n}(m)=1 /\left|Q_{n}(m)\right|$, where

$$
Q_{n}(m)=\prod_{s=1}^{m}\left[\varepsilon_{n+s}(\alpha)-\varepsilon_{n}(\alpha)\right]
$$

From Eq. A2,$Q_{n}(m)$ is a polynomial in $\alpha$.

For a polynomial $P(\alpha)$ we define by lowdeg $P(\alpha)$ the multiplicity of the root $\alpha=0$, i.e., the lowest power of $\alpha$ in the polynomial. The exponent $\nu$ that characterizes the decay of $K_{n}(m)$ (6) is given by $\nu=\operatorname{lowdeg} Q_{n}(m) / m$ for $m \rightarrow \infty$.

The data of the numerical experiments presented in Fig. [2] show that $0.894<\operatorname{lowdeg} Q_{n}(m) / m<1.12$ independent of $n$ for large $m$.

To obtain an analytical estimate we rewrite Eq. A3 as

$$
\operatorname{lowdeg} Q_{n}(m)=\sum_{s=1}^{m} \operatorname{lowdeg}\left[\varepsilon_{n+s}(\alpha)-\varepsilon_{n}(\alpha)\right] .
$$

Each term lowdeg $\left[\varepsilon_{n+s}(\alpha)-\varepsilon_{n}(\alpha)\right]$ is an integer between 0 and $n+1$.

In order to find bounds for $\operatorname{lowdeg} Q_{n}(m)$ we will estimate how many terms lowdeg $\left[\varepsilon_{n+s}(\alpha)-\varepsilon_{n}(\alpha)\right]$ exceed a given $i, 0 \leq i \leq n+1$. For each $i$ we have a subset $S_{n m}(i)$ of the values $s$ that satisfy this condition,

$$
S_{n m}(i)=\left\{s \mid 1 \leq s \leq m, \operatorname{lowdeg}\left[\varepsilon_{n+s}(\alpha)-\varepsilon_{n}(\alpha)\right]>i\right\} .
$$

The number of elements in $S_{n m}(i)$ is denoted by $h_{n m}(i)$. This is the number of polynomials $\varepsilon_{n+s}(\alpha)-\varepsilon_{n}(\alpha)$ whose expansion in $\alpha$ starts with $\alpha^{k}$ with $k>i$. In what follows for brevity we drop the subscripts $n, m$ and use $S(i)$ and $h(i)$ for $S_{n m}(i)$ and $h_{n m}(i)$.

It follows from the definition that

$$
h(0) \geq h(1) \geq h(2) \geq \cdots \geq h(n) .
$$

By construction

$$
\begin{aligned}
\operatorname{lowdeg} Q_{n}(m)= & \sum_{i=1}^{n} i[h(i-1)-h(i)] \\
& +(n+1) h(n)=\sum_{i=0}^{n} h(i)
\end{aligned}
$$

From Eq. (A6) we see that the upper and lower bounds on lowdeg $Q_{n}(m)$ are given by the sums of the upper and lower bounds of $h(i)$.

In what follows we will use the standard notations: liminf (limsup) means the lower (upper) limit of a sequence, $\operatorname{LCM}\left\{i_{1}, \ldots, i_{r}\right\}$ is the least common multiple of integers $i_{1}, \ldots, i_{r}$, and $\operatorname{GCD}\left\{i_{1}, \ldots, i_{r}\right\}$ is the greatest common divisor of $i_{1}, \ldots, i_{r}$. We will also denote by $[\varepsilon(\alpha)]_{k}$ the coefficient at $\alpha^{k}$ in the polynomial $\varepsilon(\alpha)$, i.e.,

$$
\varepsilon_{n}(\alpha)=\sum_{k=0}^{n}\left[\varepsilon_{n}(\alpha)\right]_{k} \alpha^{k}
$$

\section{Lower bound}

In this section we obtain the lower bound of $\operatorname{low} \operatorname{deg} Q_{n}(m)$. The main statement is the following lemma.

Lemma A.1 The lower bound has the form

$$
\liminf _{m \rightarrow \infty} \frac{\operatorname{lowdeg} Q_{n}(m)}{m} \geq 0.89 .
$$

Proof. Consider first the constant term $\left[\varepsilon_{n}(\alpha)\right]_{0}$ in Eq. (A7). By definition, $\left[\varepsilon_{n}(\alpha)\right]_{0}=\left[\varepsilon_{n+2}(\alpha)\right]_{0}$, and

$$
\operatorname{lowdeg}\left(\varepsilon_{n+s}(\alpha)-\varepsilon_{n}(\alpha)\right)= \begin{cases}0 & \text { for odd } s \\ \geq 1 & \text { for even } s .\end{cases}
$$

Hence, we immediately obtain a simple lower bound lowdeg $Q_{n}(m) \geq\lfloor m / 2\rfloor$ for large $m$ (in what follows we always imply $m \rightarrow \infty)$.

We compute $h(0), h(1)$, etc, using that the coefficients $\left[\varepsilon_{n}(\alpha)\right]_{i}$ are periodic in $n$ with period $2(i+1)$. Indeed, from Eq. (A2),

$$
\begin{aligned}
{\left[\varepsilon_{n}(\alpha)\right]_{i}=} & (-1)^{\lfloor n /(i+1)\rfloor} \\
& =(-1)^{\lfloor[n+2(i+1)] /(i+1)\rfloor}=\left[\varepsilon_{n+2(i+1)}(\alpha)\right]_{i} .
\end{aligned}
$$

Therefore, the sets of coefficients $\left\{\left[\varepsilon_{n}(\alpha)\right]_{0},\left[\varepsilon_{n}(\alpha)\right]_{1}, \ldots,\left[\varepsilon_{n}(\alpha)\right]_{i}\right\}$ are also periodic in $n$, but with the period $T_{i}=2 \operatorname{LCM}\{2,3, \ldots, i+1\}$. This is illustrated by the table

$$
\begin{aligned}
& \varepsilon_{1}(\alpha)=-1-\alpha \\
& \varepsilon_{2}(\alpha)=1+\alpha-\alpha^{2} \\
& \varepsilon_{3}(\alpha)=-1+\alpha+\alpha^{2}-\alpha^{3} \\
& \varepsilon_{4}(\alpha)=1-\alpha+\alpha^{2}+\alpha^{3}-\alpha^{4} \\
& \varepsilon_{5}(\alpha)=-1-\alpha+\alpha^{2}+\alpha^{3}+\alpha^{4}-\alpha^{5} \\
& \varepsilon_{6}(\alpha)=1+\alpha-\alpha^{2}+\alpha^{3}+\alpha^{4}+\alpha^{5}-\alpha^{6} \\
& \varepsilon_{7}(\alpha)=-1+\alpha-\alpha^{2}+\alpha^{3}+\alpha^{4}+\alpha^{5}+\alpha^{6}-\alpha^{7}
\end{aligned}
$$

In order to estimate $h(i)$ we need two technical statements.

Lemma A.2 Let $a_{0}, k, T$ be any integers such that $2 k$ does not divide $T$. Consider any $2 k / \operatorname{GCD}\{T, 2 k\}$ consecutive elements of an arithmetic progression $a_{j}=$ $a_{0}+j \cdot T$, and set $b_{j}=\left\lfloor a_{j} / k\right\rfloor \bmod 2$.

Then, at least $\lfloor k / \operatorname{GCD}\{T, 2 k\}\rfloor$ integers $b_{j}$ are equal to 0 , and at least the same number of $b_{j}$ are equal to 1 .

Proof. Since $2 k$ does not divide $T$, sequence $a_{j} \bmod 2 k$ is cyclic in the interval $[0,2 k-1]$. This sequence contains exactly $2 k / \operatorname{GCD}\{T, 2 k\}$ distinct elements. On average, half of them (at least $\lfloor p / \operatorname{GCD}\{T, 2 k\}\rfloor$ ) are less than $p$, and another half (the same number) are larger or equal than $p$. This means that there are at least $\lfloor k / \operatorname{GCD}\{T, 2 k\}\rfloor$ integers $b_{j}$ that are equal to 0 and at least the same number of $b_{j}$ that are equal to 1. Q.E.D.

The next statement is a corollary of lemma A.2 and we skip the proof. 
Corollary A.3 Let $2 k$ does not divide T. Consider $p$ consecutive elements of the arithmetic progression $a_{j}=$ $a_{0}+j \cdot T$ and set $b_{j}=\left\lfloor a_{j} / k\right\rfloor \bmod 2$.

Then at least $\lfloor k / \mathrm{GCD}\{T, 2 k\}\rfloor\lfloor p \cdot \operatorname{GCD}\{T, 2 k\} / 2 k\rfloor$ integers $b_{j}$ are equal to 0 , and at least the same number of $b_{j}$ are equal to 1.

We are now in a position to finish the proof of Lemma A.1 We notice first that, for $n=a_{j}$ in the expression (A2), the coefficient $b_{j}$ for given $k$ determines the sign of the term $\alpha^{k-1}$ in $\varepsilon_{n}(\alpha)$, that is $(-1)^{b_{j}}=$ $\left[\varepsilon_{n}(\alpha)\right]_{k-1}$. The number $h(i)$ gives the probability that, for all $k \leq i$, the polynomial $\varepsilon_{n+s}(\alpha)$ has the same $b_{j}$ as $\varepsilon_{n}(\alpha)$.

We will now estimate $h(i)$ with $i=1, \ldots, 4$ and start with $h(1)$. We note that $s \in S(1)$ if and only if $s \in S(0)$ and $\left[\varepsilon_{n+s}\right]_{1}=\left[\varepsilon_{n}\right]_{1}$. The second condition means that $\lfloor s / 2\rfloor \bmod 2=0$. By construction A5, for $m \rightarrow \infty$ the set $S(0)$ is formed by all numbers $s$ of the same parity as $n$. This means that $S(0)$ is an arithmetic progression with period $T_{0}=2$. We take $p$ consecutive elements $s_{1}, \ldots s_{p}$ of it and use Corollary A.3 with $k=2$, because we are interested in the coefficients $\left[\varepsilon_{n+s_{i}}\right]_{1}=(-1)^{\left\lfloor\left(n+s_{i}\right) / 2\right\rfloor}$ in A2 . By Corollary A.3. since $T_{0}$ is not divisible by $2 k$, for at least $\lfloor p / 2\rfloor$ subscripts $s_{i}$ the coefficients $\left[\varepsilon_{n+s_{i}}(\alpha)\right]_{1}=1$, and $\left[\varepsilon_{n+s_{i}}(\alpha)\right]_{1}=-1$ for at least the same amount of subscripts $s_{i}$, i.e., approximately half of the coefficients $\left[\varepsilon_{n+s_{i}}(\alpha)\right]_{1}$ coincide with $\left[\varepsilon_{n}(\alpha)\right]_{1}$. Hence, $h(1) \geq h(0) / 2$ as $m \rightarrow \infty$. Substituting $h(0)=m / 2$ we obtain $h(1)=m / 4$.

Similar arguments can be applied to estimate $h(2)$. This requires finding a portion of the set $S(1)$ which forms $S(2)$. The set $S(1)$ is a nonempty disjoint union of arithmetic progressions with period $T_{1}=4$. We will apply Corollary A.3 with $k=3$ to each of these progressions and use $p=h(1)$. This gives $h(2) \geq$ $\lfloor 3 / 2\rfloor\lfloor(1 / 3)(m / 4)\rfloor=\lfloor m / 12\rfloor$, or $h(2) / m \geq 1 / 12$ for $m \rightarrow \infty$.

In the same way we obtain $h(3) / m \geq 1 / 24$ and $h(4) / m \geq 1 / 60$ as $m \rightarrow \infty$. Therefore

$$
\operatorname{lowdeg} Q_{n}(m) / m \geq \frac{1}{2}+\frac{1}{4}+\frac{1}{12}+\frac{1}{24}+\frac{1}{60} \geq 0.89,
$$

which finishes the proof of the lower bound.

\section{Upper bound}

We start with the proof of the following rough estimate:

Lemma A.4 An upper bound has the form

$$
\limsup _{n \rightarrow \infty} \frac{\operatorname{lowdeg} Q_{n}(m)}{m} \leq \frac{22}{15}<1.47 .
$$

Proof. Taking in the rhs of Eq. A6 the sum from 0 to $\infty$ we obtain

$$
\operatorname{lowdeg} Q_{n}(m) \leq \sum_{i=0}^{\infty} h(i) .
$$

To find an upper bound on $h(i)$ we will use the following consequence of Lemma A.2

Corollary A.5 Let $2 k$ does not divide T. Consider $p$ consecutive elements of the arithmetic progression $a_{j}=$ $a_{0}+j \cdot T$ and set $b_{j}=\left\lfloor a_{j} / k\right\rfloor \bmod 2$.

Then at most

$$
\begin{aligned}
& \left(\frac{2 k}{\operatorname{GCD}\{T, 2 k\}}-\left\lfloor\frac{k}{\operatorname{GCD}\{T, 2 k\}}\right\rfloor\right) \\
& \times\left(\left\lfloor\frac{p \cdot \operatorname{GCD}\{T, 2 k\}}{2 k}\right\rfloor+1\right)
\end{aligned}
$$

integers $b_{j}$ are equal to 0 , and at most the same number of $b_{j}$ are equal to 1 .

Using the same arguments as before, by corollary A.5 we obtain for $m \rightarrow \infty$ the following upper bounds for $h(i)$

$$
\begin{aligned}
h(0) & \leq m / 2, \\
h(1) & \leq m / 4, \\
h(2) & \leq m / 6, \\
h(3) & \leq m / 12 \\
h(4) & \leq m / 20 .
\end{aligned}
$$

Recall that $h(4) \geq h(5) \geq h(6) \geq h(7)$. Similarly, for $q \geq 2$ we have

$$
h\left(2^{q}\right) \geq h\left(2^{q}+1\right) \geq \ldots h\left(2^{q+1}-1\right) .
$$

Therefore we can replace the terms $h\left(2^{q}+1\right), \ldots, h\left(2^{q+1}-1\right)$ in the rhs of Eq. A8 by $h\left(2^{q}\right)$, which leads to the following upper bound for lowdeg $Q_{n}(m)$,

$\operatorname{lowdeg} Q_{n}(m) \leq h(0)+h(1)+h(2)+h(3)+\sum_{q=2}^{\infty} 2^{q} h\left(2^{q}\right)$.

This reduces the calculation to finding upper bounds on $h\left(2^{q}\right)$.

We will now obtain a recurrence relation for $h\left(2^{q}\right)$. First we notice that, for $K+1$ being a prime number, we have from Corollary A.5

$$
h(K+1) \leq \frac{K+2}{2(K+1)} h(K) .
$$

For all primes $K+1 \geq 7$ we have $(K+2) / 2(K+1) \leq 4 / 7$. Therefore $h(K+1) \leq \frac{4}{7} h(K)$ for $K \geq 6$. We also note that, for all positive integral $q$,

$$
h\left(2^{q}\right) \leq \frac{1}{2} h\left(2^{q}-1\right) .
$$

Now we recall the distribution law for primes in the intervals. The following statement is called Bertrand's postulate (or Tchebychev' theorem) (see Ref. 22): 
Theorem A.6 There is at least one prime between $M$ and $2 M$ for any positive integer $M$. If $M>3$, there is always at least one prime between $M$ and $2 M-2$.

In particular, there is at least one prime between $2^{q}$ and $2^{q+1}-1$ for any positive integer $q \geq 2$. With this statement, taking into account the previous estimates, we obtain

$$
h\left(2^{q+2}\right) \leq\left(\frac{1}{2} \cdot \frac{4}{7}\right)^{q} \frac{m}{20}
$$

for all positive integral $q$, or

$$
h\left(2^{q+2}\right) \leq\left(\frac{2}{7}\right)^{q} \frac{m}{20} .
$$

Substituting inequalities (A9), A12 into A10, we obtain

$$
\begin{aligned}
\operatorname{lowdeg} Q_{n}(m) & \leq \frac{m}{2}+\frac{m}{4}+\frac{m}{6}+\frac{m}{12}+\frac{m}{20}\left(2^{2}+2^{3} \cdot \frac{2}{7}+2^{4}\left(\frac{2}{7}\right)^{2}+\ldots+2^{k}\left(\frac{2}{7}\right)^{k-2}+\ldots\right) \\
& =m\left(1+\frac{2^{2}}{20} \sum_{j=0}^{\infty}\left(\frac{4}{7}\right)^{j}\right) \quad \text { for } \quad m \rightarrow \infty .
\end{aligned}
$$

This gives

$$
\operatorname{lowdeg} Q_{n}(m) \leq \frac{22}{15} m<1.47 m
$$

The last inequality is an explicit asymptotic upper bound. Q.E.D.

We now provide a sharper upper bound. We will use the same method, but instead of Tchebyshev' theorem we will apply Erdös theorem.

Lemma A.7 (Sharper bound)

$$
\limsup _{m \rightarrow \infty} \frac{\operatorname{lowdeg} Q_{n}(m)}{m} \leq 1.19
$$

Proof. Following the same pattern as in Lemma A.4 above we extend the explicit list of inequalities (A9).

As $m \rightarrow \infty$,

$$
\begin{aligned}
h(5) / m & \leq 1 / 20, \\
h(6) / m & \leq 1 / 35, \\
h(7) / m & \leq 1 / 70, \\
h(8) / m & \leq 1 / 126, \\
h(9) / m & \leq 1 / 126, \\
h(10) / m & \leq 1 / 231, \\
h(11) / m & \leq 1 / 231, \\
h(12) / m & \leq 1 / 429, \\
h(13) / m & \leq 1 / 429, \\
h(14) / m & \leq 1 / 429, \\
h(15) / m & \leq 1 / 858, \\
h(16) / m & \leq 3 / 4862 .
\end{aligned}
$$

To obtain a sharper upper bound we recall the following result by Erdös [23].
Theorem A.8 (Erdös) There exist at least one prime of the form $4 k+1$ and at least one prime of the form $4 k+3$ between $M$ and $2 M$ for all $m>6$.

For all primes that exceed 16 we have in Eq. A11 $(K+2) / 2(K+1)<7 / 13$. Therefore, by reproducing the arguments that led to the inequality A12, but using now the relation (A11) twice based on the theorem A.8 we obtain

$$
h\left(2^{q+4}\right) \leq\left[\frac{1}{2} \cdot\left(\frac{7}{13}\right)^{2}\right]^{q} h(16) .
$$

Substituting inequalities (A9), A14, A15 into (A10) (where now the terms up to $h(15)$ are taken into account explicitly, and the sum runs from $q=4$ ) we obtain

$$
\begin{aligned}
& \limsup _{m \rightarrow \infty} \frac{\operatorname{lowdeg} Q_{n, m}}{m} \leq \frac{1}{2}+\frac{1}{4}+\frac{1}{6}+\frac{1}{12}+\frac{2}{20} \\
& +\frac{1}{35}+\frac{1}{70}+\frac{2}{126}+\frac{2}{231}+\frac{3}{429}+\frac{1}{858} \\
& +\frac{3 \cdot 16}{4862}\left(1+\left(\frac{7}{13}\right)^{2}+\left(\frac{7}{13}\right)^{4}+\ldots\right) .
\end{aligned}
$$

Evaluating the rhs, we obtain

$$
\limsup _{m \rightarrow \infty} \frac{\operatorname{lowdeg} Q_{n}(m)}{m}<1.19 .
$$

Q.E.D. 


\section{APPENDIX B: THE INTERRELATION BETWEEN THE ENERGY SPECTRUM PARAMETERS $\alpha$ AND $J / h$ FOR FIXED IPR}

In this Appendix we outline another way of looking at the effect of the band structure of sequence (4) on localization. It applies to single-particle stationary states and is based on varying $h / J$ and finding such energy spectrum parameter $\alpha$ that would keep the IPR constant, i.e.,

$$
\left\langle I_{1}\right\rangle \equiv\left\langle I_{1}(\alpha, J / h)\right\rangle=\text { const. }
$$

The average IPR is large, $\approx L / 3$, when the spread of the on-site energies $\alpha h$ is small compared to the hoppinginduced bandwidth $J^{2} / 2 h$ of the bands at $\pm h / 2$. When $\alpha h$ becomes comparable to $J^{2} / h$, a part of the states become localized with localization length smaller than the chain size, but still there remain states of size $\sim L$. For such states $I_{1 \lambda} \propto L$. Their portion depends on $\alpha h /\left(J^{2} / h\right)$. Therefore one may expect that, for large $\left\langle I_{1}\right\rangle$ and for a given chain length, $\alpha$ should vary with $J / h$ as $(J / h)^{2}$.

Another scaling region of $\alpha(J / h)$ as given by Eq. (B1) may be expected to emerge for $\alpha$ close to the threshold value, $\alpha_{\text {th }} \lesssim \alpha \ll 1$, but far away from the stronglocalization range of $\alpha$, where $\left\langle I_{1}\right\rangle-1 \sim J^{2} / h^{2}$ [cf. Eq. (7)].

For $\alpha$ close to $\alpha_{\mathrm{th}}$, the wave functions have comparatively small-amplitude tails that spread over a long distance and are nearly exponential at large distances, as given by Eq. (6). If the decay were purely exponential, i.e., the tail of the wave function centered on site $n$ were of the form $\psi_{n+m}=K^{|m|} \psi_{n}$, we would have $\left\langle I_{1}\right\rangle-1 \approx 4|K|^{2}$ for $K \approx J / 2 \alpha h \ll 1$. From (B1), this condition gives scaling $\alpha \propto J / h$. The nonexponential decay of the wave functions at small to moderate distances (numerically, for $|m| \sim 4-8$ ) leads to deviations from this simple scaling.

Numerical results on the dependence of $\alpha$ on $J / h$ as given by Eq. (B1) are shown in Fig. 10 The data for $\left\langle I_{1}\right\rangle \sim L / 3$ show the expected scaling $\alpha \propto(J / h)^{2}$. On the other hand, in the range $\left\langle I_{1}\right\rangle-1 \approx 0.1-1$ the value of $\alpha$ scales as $J / h$. This scaling applies only for $\alpha>\alpha_{\mathrm{th}}$, i.e., for $\alpha h / J>1 / 2$. The value of $\alpha h / J$ as given by Eq. (B1) increases with decreasing $\left\langle I_{1}\right\rangle$.

We note that, for large $h / J \sim 100$ and small $\left\langle I_{1}\right\rangle-1$, the IPR $\left\langle I_{1}\right\rangle$ as a function of $\alpha$ displays small oscillations. This leads to multivaluedness of the roots $\alpha$ of the equation $\left\langle I_{1}\right\rangle=$ const. The roots are numerically very close to each other. We showed the multivaluedness schematically by plotting $\alpha$ vs. $h / J$ in Fig. 10 with jagged lines.

In the intermediate range of $\left\langle I_{1}\right\rangle$, the function $\alpha(J / h)$ crosses over from one type of the limiting behavior to the other. The numerical data does not seem to suggest that $\left\langle I_{1}\right\rangle$ has a universal scaling form of a function of $\alpha^{\nu} /(J / h)$ for all $\alpha, J / h \ll 1$.
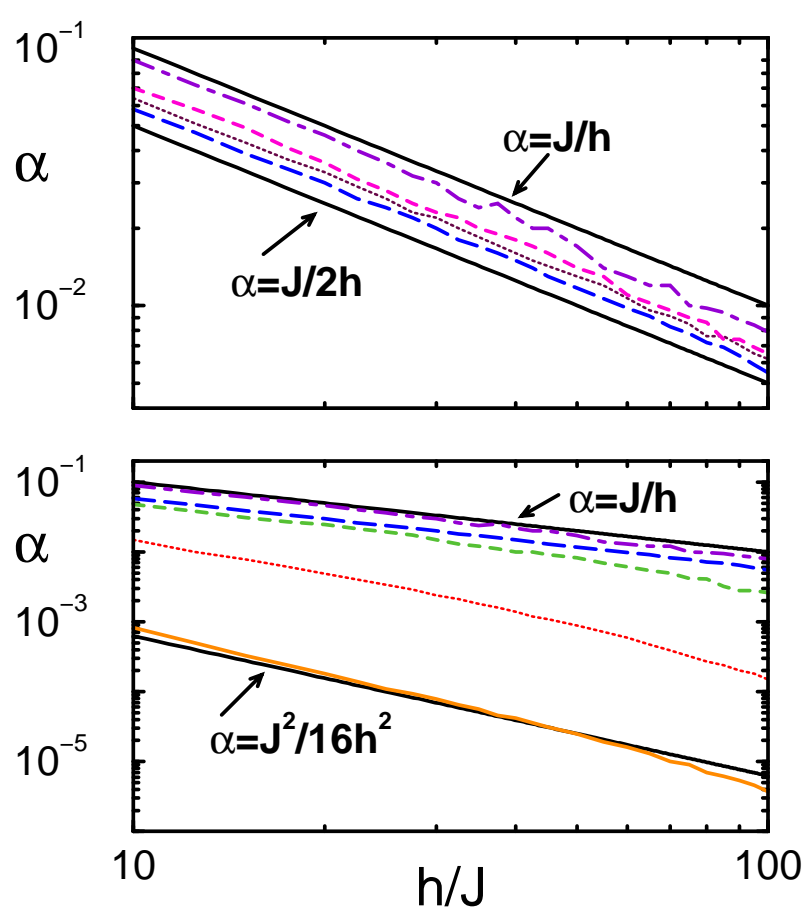

FIG. 10: (color online). The dependence of $\alpha$ on $h / J$ as given by the condition $\left\langle I_{1}\right\rangle=$ const for different $\left\langle I_{1}\right\rangle$ in the chain with $L=300$. The lines in the lower panel listed from down upward (thin solid, dotted, dashed, long-dashed, and dot-dashed) correspond to $\left\langle I_{1}\right\rangle=95,50,10,2$, and 1.2, respectively. The lines in the upper panel listed from down upwards (long-dashed, dotted, dashed, and dot-dashed) correspond to comparatively small $\left\langle I_{1}\right\rangle=2,1.6,1.4$, and 1.2 , respectively. The bold lines $\alpha=J^{2} / 16 h^{2}$ and $\alpha=J / h$ display the asymptotic behavior of $\alpha$ for large and small $\left\langle I_{1}\right\rangle$; the line $\alpha=J / 2 h$ corresponds to $\alpha=\alpha_{\text {th }}$.

\section{APPENDIX C: NARROW RESONANCES OF THE MANY-PARTICLE IPR AS FUNCTION OF THE PARAMETER $\alpha$}

In this Appendix we discuss the positions and widths of the narrow peaks of the IPR seen in Fig. 4

As we increase $\alpha$ starting from $\alpha=0$, pronounced peaks of $\left\langle I_{6}\right\rangle$ appear for the difference in the combination two-particle on-site energies $\delta \varepsilon=\mid \varepsilon_{k_{1}}+\varepsilon_{k_{2}}-\varepsilon_{k_{3}}-\varepsilon_{k_{4}}$ (15) equal to

$$
\delta \varepsilon \approx s \alpha h \approx J \Delta
$$

with $s=1,2$. They are due to resonant hybridization of pairs on neighboring sites $(n, n+1)$ with dissociated pairs located on sites $(n, n+3)$ for $s=1$, and $(n-1, n+2)$ with even $n$ for $s=2$, for example. Such hybridization corresponds to two single-particle steps by one site, i.e. $\varkappa=2$.

A specific example for the studied chain with $\Delta=1$, $h / J=20$, and $\alpha=0.05$ is the resonance between the on-site states $|\Phi(3,4,6,7,8,9)\rangle,|\Phi(4,5,6,7,8,9)\rangle$, $|\Phi(3,4,6,7,8,11)\rangle$ and $|\Phi(4,5,6,7,8,11)\rangle$ (we remind 
that the arguments of $\Phi$ indicate the positions of the excitations; we have six excitations, and the available sites are $1,2, \ldots, 12)$. All these states can be obtained from each other by moving one excitation by two positions. For example, in the first pair the excitation goes from site 3 , where it has one nearest neighbors, to site 5 , where it has two neighbors.

The width of the above peaks $\delta \alpha$ can be estimated from the condition that the frequency detuning $|s \alpha h \pm J \Delta|$ is of order of the effective hopping integral $J \Delta V_{k_{1} k_{2} k_{3} k_{4}}$. For $s=1$ [an $(n, n+1) \leftrightarrow(n, n+3)$-type transition] the hopping integral is $\sim J^{3} \Delta / \alpha h^{2}$ from Eqs. (11), (12). This gives the width

$$
\delta \alpha \sim(J / h)^{2} .
$$

The positions of the peaks $\alpha \approx J \Delta / s h$ and their widths are in agreement with the data in both upper and lower main panels of Fig. [4

For larger $\alpha$, narrow resonances with respect to $\alpha$ occur when

$$
s \alpha^{m} h \approx M J \Delta
$$

with integer $s, m, M$, and $m \geq 2, M=1,2$. They may happen, for example, between pairs $(n, n+1)$ and $(n-1, n+2)$ with odd $n$ such that $n \neq 3 k-1$, in which case $m=2$ and $\varkappa=2$. A specific example for our chain is the resonance between the on-site states $|\Phi(1,3,4,6,9,11)\rangle$ and $|\Phi(1,2,5,6,9,11)\rangle$ for $\Delta=$ $1, h / J=20$, and $\alpha=0.246$. Here the excitations on sites $(3,4)$ move to sites $(2,5)$, and $\alpha^{2} h \sim J \Delta$ (in fact, higherorder terms in $\alpha$ are essential for fine-tuning the states into resonance). In other cases resonances with $m \geq 2$ require more intermediate virtual steps, with $\varkappa \geq 4$.

The $m \geq 2$-resonances are extremely narrow for $\alpha_{\mathrm{th}} \ll$ $\alpha \ll 1$. For example, for $m=2$ their widths are

$$
\begin{array}{ll}
\delta \alpha \lesssim(J / h)^{5 / 2} \Delta^{1 / 2} & \text { for } \varkappa=2, \\
\delta \alpha \lesssim J^{3} / h^{3} \Delta & \text { for } \varkappa=4 .
\end{array}
$$

In these estimates we used that, from Eqs. (41), (12) $\left|V_{n-1 n+2 n n+1}\right| \lesssim J^{2} / h^{2}$ for the $\varkappa=2$-transition $(n, n+$ $1) \leftrightarrow(n-1, n+2)$. For the $m=2$ and $\varkappa=4$-transitions, on the other hand, $\left|V_{k_{1} k_{2} k_{3} k_{4}}\right| \lesssim J^{4} / \alpha^{3} h^{4}$ [for example, this estimate applies to a transition $(n, n+1) \leftrightarrow$ $(n, n+5)]$. We note that, from the condition $\alpha_{\text {th }} \ll \alpha$ and the resonance condition $s \alpha^{2} h=M J \Delta$, it follows that $\Delta \gg J / h$, which guarantees the smallness of the peak widths.

Each high-order resonance gives rise to a narrow band of resonant $\alpha$ values. All of them refer to a resonant transition between the same sites. However, the energy difference of these sites is slightly different depending on the occupation of remote sites, for example, next nearest neighbors. In this latter case, from Eqs. (11), (12), the corresponding shift of $\alpha$ is $\propto(J / h)^{5 / 2} \Delta^{1 / 2}$.

A specific example for the studied chain is provided by the resonances between two pairs of on-site states, $|\Phi(2,3,6,7,8,12)\rangle$ and $|\Phi(2,3,6,8,11,12)\rangle$, on the one hand, and $|\Phi(5,6,7,8,9,12)\rangle$ and $|\Phi(5,6,8,9,11,12)\rangle$, on the other hand. In both cases the resonant transition is fermion hopping from site 7 to site 11. Both resonances occur for $\Delta=1, h / J=20$, but the first corresponds to $\alpha \approx 0.2778$, whereas the second corresponds to $\alpha \approx$ 0.2782 . The difference in $\alpha$ comes primarily from the different occupation of the next nearest neighbors of sites 7 and 11.

The most pronounced peaks in Fig. 4 correspond to comparatively small $\varkappa \leq 4$. However, there are resonances for higher $\varkappa$ as well. The resonating energies have to be extremely close to each other for such states to be hybridized. The corresponding peaks are very narrow, and very high precision is needed to find them numerically (sometimes the hybridization appears to be an artifact of not sufficiently precise calculations).

As mentioned above, in the region $0.2 \lesssim \alpha \lesssim 0.4$ the positions of the IPR peaks are determined not only by the leading-order terms in $\alpha$, but also by higher-order terms. Therefore there are several resonant bands for each $s, m, M$ as given by the condition $s \alpha^{m} h \approx M J \Delta$. This explains why there are several bands in Fig. [4 These bands are well separated for sufficiently large $h / J$ and not too large $\Delta$. On the other hand, for $h / J=10$ and $\Delta=3$ the bands of resonances are broadened and overlap with each other. When $\alpha$ is not very small there emerge also narrow resonances where $\delta \varepsilon \ll J$ and $\delta \varepsilon / h \rightarrow 0$. They are responsible for some of the peaks in the insets of Fig. 目
[1] P. W. Anderson, Phys. Rev. 109, 1492 (1958).

[2] C. W. J. Beenakker, Rev. Mod. Phys. 69, 731 (1997).

[3] B. Kramer and A. MacKinnon, Rep. Prog. Phys. 56, 1469 (1993).

[4] M. A. Nielsen and I. L. Chuang, Quantum Computation and Quantum Information (Cambridge University Press, Cambridge, 2000); L.M.K. Vandersypen and I.L. Chuang, quant-ph/0404064

[5] Y. Makhlin, G. Schön, and A. Shnirman, Rev. Mod. Phys. 73, 357 (2001).
[6] P. M. Platzman and M. I. Dykman, Science 284, 1967 (1999).

[7] J. E. Mooij, T. P. Orlando, L. Levitov, L. Tian, C. H. van der Wal, and S. Lloyd, Science 285, 1036 (1999).

[8] T. D. Ladd, J. R. Goldman, F. Yamaguchi, Y. Yamamoto, E. Abe, and K. M. Itoh, Phys. Rev. Lett. 89, 017901 (2002).

[9] Yu. A. Pashkin, T. Yamamoto, O. Astafiev, Y. Nakamura, D. V. Averin, and J. S. Tsai, Nature 421, 823 (2003). 
[10] W. G. Van der Wiel, S. De Franceschi, J. M. Elzerman, T. Fujisawa, S. Tarucha, and L. P. Kouwenhoven, Rev. Mod. Phys. 75, 1 (2003).

[11] X. Zhou, Z. Zhou, G. Guo, and M. J. Feldman, Phys. Rev. Lett. 89, 197903 (2002).

[12] S. C. Benjamin and S. Bose, Phys. Rev. Lett. 90, 247901 (2003).

[13] G. P. Berman, F. Borgonovi, F. M. Izrailev, and V. I. Tsifrinovich, Phys. Rev. E 64, 056226 (2001); 65, 015204 (2002).

[14] R. Berkovits, Y. Gefen, I. V. Lerner, and B. L. Altshuler, Phys. Rev. B 68, 085314 (2003); B.L. Altshuler, Y. Gefen, A. Kamenev, and L. S. Levitov, Phys. Rev. Lett. 78, 2803 (1997) and references therein.

[15] B. Georgeot and D. L. Shepelyansky, Phys. Rev. E 62,
3504, 6366 (2000).

[16] M. I. Dykman and P. M. Platzman, Fortschr. Phys. 48, 9 (2000); Quantum Inf. Comput. 1, 102 (2001).

[17] D. J. Thouless and Q. Niu, J. Phys. A 16, 1911 (1983).

[18] J. B. Sokoloff, Phys. Rep. 126, 189 (1985).

[19] E. L. Albuquerque and M. G. Cottam, Phys. Rep. 376, 225 (2003).

[20] R. Merlin, K. Bajema, R. Clarke, F.-Y. Juang, and P.K. Bhattacharya, Phys. Rev. Lett. 55, 1768 (1985).

[21] P. Jordan and E. Wigner, Z. Phys. 47, 631 (1928).

[22] T. Nagell, Introduction to Number Theory (Wiley, New York 1951).

[23] P. Erdös, J. London Math. Soc. 9, 282 (1934). 